\title{
Diastereoselective Synthesis of Spiro[pyrazolone-4,3'-tetrahydro- thiophenes] via a Sulfa-Michael/Aldol Domino Reaction
}

\author{
Uğur Kayaa \\ Suruchi Mahajan ${ }^{a}$ \\ Jan-Hendrik Schöbel ${ }^{\mathrm{a}}$ \\ Arto Valkonen ${ }^{\mathrm{b}}$ \\ Kari Rissanen ${ }^{b}$ \\ Dieter Enders*a \\ a Institute of Organic Chemistry, RWTH Aachen University, \\ Landoltweg 1, 52074 Aachen, Germany \\ enders@rwth-aachen.de \\ b Department of Chemistry, Nanoscience Center, University \\ of Jyvaskyla, 40014 JYU, Finland
}

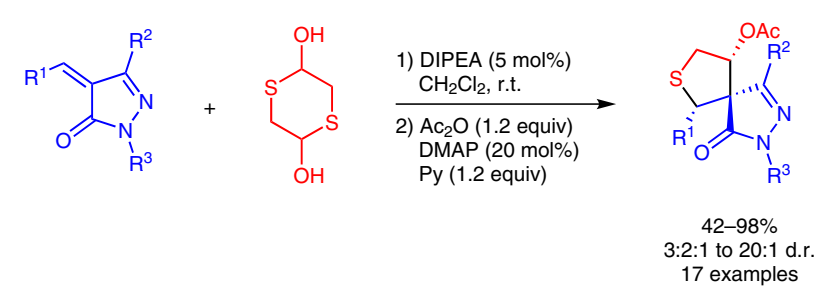

Received: 14.06.2016

Accepted: 14.06.2016

Published online: 12.08 .2016

DOI: 10.1055/s-0035-1562473; Art ID: ss-2016-z0427-op

License terms: (C) (1)

Abstract A new approach for the diastereoselective synthesis of spiro[pyrazolone-4,3'-tetrahydrothiophenes] was developed. The $\mathrm{N}, \mathrm{N}$ diisopropylethylamine-catalyzed reaction of arylidenepyrazolones with in situ generated 2-sulfanylacetaldehyde provides the corresponding spiro-heterocycles via a domino sulfa-Michael/aldol reaction in 42-98\% yield and 3:2:1 to 20:1 d.r. under mild reaction conditions.

Key words spiro compounds, domino reaction, tetrahydrothiophene, pyrazolone, sulfa-Michael addition

The development of new and efficient synthetic routes to access bioactive heterocyclic compounds is fundamental for the synthesis of valuable natural products, pharmaceuticals and agrochemicals. Tetrahydrothiophenes are present in many natural products and pharmaceuticals, ${ }^{1}$ such as the essential coenzyme biotin ${ }^{2}$ or analogues of penicillin. ${ }^{3}$ In addition, bioactive compounds bearing a pyrazolone moiety display a wide range of biological and pharmaceutical properties, and especially spiro-pyrazolones, ${ }^{4}$ such as the spiro compounds $\mathbf{A},{ }^{5}$ with antimicrobial activity, $\mathbf{B}$, a phosphodiesterase inhibitor, ${ }^{6} \mathbf{C}$, with antitumor activity, ${ }^{7}$ and D, with antibacterial activity, ${ }^{8}$ have attracted much interest in medicinal chemistry (Figure 1).

Recently, arylidenepyrazolones have been recognized as very good substrates to provide new pyrazole and pyrazolone derivatives..$^{9}$ 1,4-Dithiane-2,5-diol, the dimer of 2sulfanylacetaldehyde, emerged as an excellent precursor for the development of domino sulfa-Michael/aldol reactions to provide tetrahydrothiophene rings (Scheme 1$).{ }^{10}$ Similar domino transformations of 1,4-dithiane-2,5-diol resulted in the construction of spiro-tetrahydrothiophenes containing
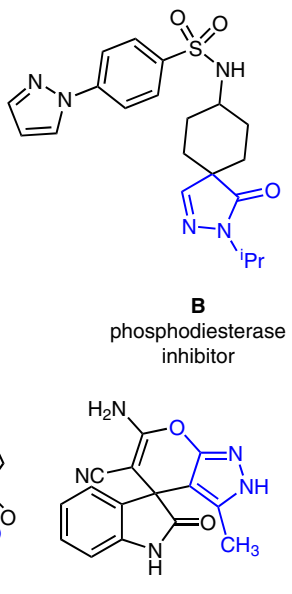

antibacterial

Figure 1 Typical examples of bioactive spiro-pyrazolone compounds

oxindole, ${ }^{11 \mathrm{a}, \mathrm{c}}$ 1,3-indanedione, ${ }^{11 \mathrm{e}}$ and chromanone cores. ${ }^{11 \mathrm{~b}, \mathrm{~d}}$ However, to the best of our knowledge, there is no report in the literature on the formation of spiro-tetrahydrothiophenes bearing a pyrazolone moiety.

Herein we report a diastereoselective sulfa-Michael/ aldol domino reaction of arylidenepyrazolones 1 and 1,4dithiane-2,5-diol (2) to provide spiro[pyrazolone-4,3'-tetrahydrothiophenes] 3 (Scheme 1). In our initial investigations, different organic and inorganic bases were tested (Table 1). The first attempt using DMAP (10 mol\%) in $\mathrm{CH}_{2} \mathrm{Cl}_{2}$ at room temperature afforded the spirocyclic product $3 \mathbf{a}$ in $83 \%$ yield and 12:2:1 d.r (entry 1). The product was acetylated in situ to ease the analysis of the NMR spectra. The screening of other organic bases such as $\mathrm{Et}_{3} \mathrm{~N}$, DABCO, or DBU provided $3 \mathbf{a}$ in moderate to very good yields and good diastereomeric ratios, but the reaction time extended to 19 hours when the base DABCO was used (entries 2-4). The sulfa- 


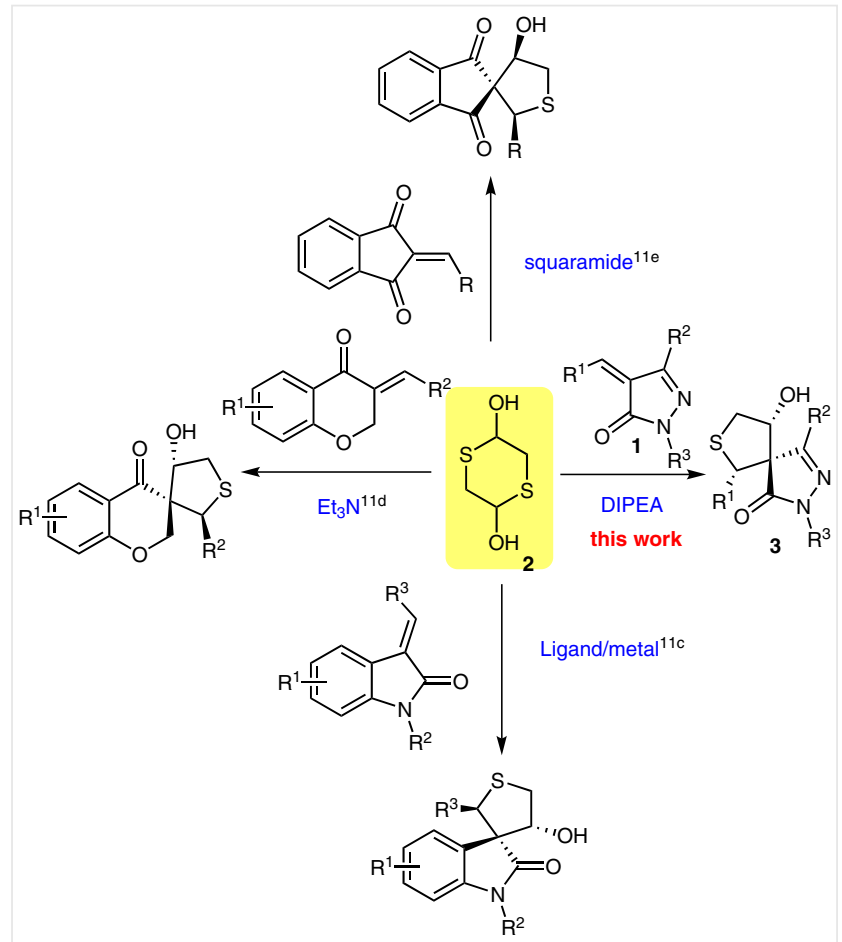

Scheme 1 Synthetic catalytic approaches to spirocyclic tetrahydrothiophenes

Michael/aldol domino reaction proceeded well when the inorganic base $\mathrm{K}_{2} \mathrm{CO}_{3}$ was used, to afford 3a in 97\% yield and 9:2:1 d.r., but in a longer reaction time (entry 5).

After further screening of organic bases, DIPEA was found to be the best catalyst, providing the spirocyclic product 3a in $98 \%$ yield and 12:2:1 d.r. within 15 minutes (Table 1 , entry 9 ). The reaction was further optimized by conducting the reaction in different solvents and varying the catalyst loading to determine the optimal reaction conditions. We found that DIPEA (5 mol\%) in $\mathrm{CH}_{2} \mathrm{Cl}_{2}$ at room temperature provided $3 a$ in the best yield of $98 \%$ and a d.r. of 15:2:1 within 15 minutes (entry 16).

The optimized reaction conditions were then used to probe the general applicability of the domino sequence (Scheme 2). The sulfa-Michael/aldol reaction of arylidenepyrazolones bearing electron-donating ( $\mathbf{1 a}-\mathbf{f})$ and electronwithdrawing groups $(\mathbf{1 g}-\mathbf{j})$ provided the spirocyclic compounds $\mathbf{3 a}-\mathbf{j}$ in good to excellent yields and moderate to excellent diastereomeric ratios. Good results in terms of yield and d.r. were also obtained by using a thionyl substituent at the arylidenepyrazolone, showing the presence of heterocyclic substituents in this reaction. The substrate scope was extended by varying the substituents at the $\mathrm{N}-1$ and $\mathrm{C}-3$ positions of the alkylidenepyrazolone. The reaction with different aryl substituents at the $\mathrm{N}-1$ position provided good yields and moderate to very good diastereomeric ratios (31-n). Moderate to good yields and moderate to excel-
Table 1 Catalyst and Solvent Screening ${ }^{a}$

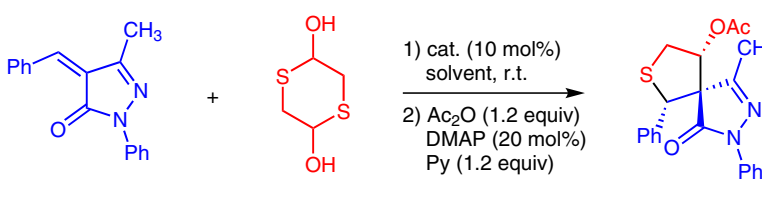

$1 \mathrm{a}$

2

rac-3a

\begin{tabular}{|c|c|c|c|c|c|}
\hline Entry & Catalyst & Solvent & Reaction time & Yield (\%) & d.r. ${ }^{c}$ \\
\hline 1 & DMAP & $\mathrm{CH}_{2} \mathrm{Cl}_{2}$ & $55 \mathrm{~min}$ & 83 & $12: 2: 1$ \\
\hline 2 & $\mathrm{Et}_{3} \mathrm{~N}$ & $\mathrm{CH}_{2} \mathrm{Cl}_{2}$ & $50 \mathrm{~min}$ & 73 & $7: 2: 1$ \\
\hline 3 & DABCO & $\mathrm{CH}_{2} \mathrm{Cl}_{2}$ & $19 \mathrm{~h}$ & 93 & $10: 2: 1$ \\
\hline 4 & DBU & $\mathrm{CH}_{2} \mathrm{Cl}_{2}$ & $90 \mathrm{~min}$ & 81 & $12: 2: 1$ \\
\hline 5 & $\mathrm{~K}_{2} \mathrm{CO}_{3}$ & $\mathrm{CH}_{2} \mathrm{Cl}_{2}$ & $48 \mathrm{~h}$ & 97 & $9: 2: 1$ \\
\hline 6 & DBN & $\mathrm{CH}_{2} \mathrm{Cl}_{2}$ & $3.5 \mathrm{~h}$ & 83 & $11: 2: 1$ \\
\hline 7 & $\mathrm{HMTA}^{\mathrm{d}}$ & $\mathrm{CH}_{2} \mathrm{Cl}_{2}$ & $4 \mathrm{~h}$ & 77 & $13: 2: 1$ \\
\hline 8 & TMEDA & $\mathrm{CH}_{2} \mathrm{Cl}_{2}$ & $30 \mathrm{~min}$ & 98 & $12: 2: 1$ \\
\hline 9 & DIPEA & $\mathrm{CH}_{2} \mathrm{Cl}_{2}$ & $15 \mathrm{~min}$ & 98 & $12: 2: 1$ \\
\hline 10 & DIPEA & DCE & $30 \mathrm{~min}$ & 79 & $15: 2: 1$ \\
\hline 11 & DIPEA & toluene & $5 \mathrm{~h}$ & 96 & $15: 2: 1$ \\
\hline 12 & DIPEA & THF & $20 \mathrm{~min}$ & 74 & $6: 1: 1$ \\
\hline $13^{e}$ & DIPEA & $\mathrm{CH}_{2} \mathrm{Cl}_{2}$ & $7 d$ & n.d. & n.d. \\
\hline $14^{f}$ & DIPEA & $\mathrm{CH}_{2} \mathrm{Cl}_{2}$ & $20 \min$ & 37 & $17: 2: 1$ \\
\hline $15^{g}$ & DIPEA & $\mathrm{CH}_{2} \mathrm{Cl}_{2}$ & $25 \min$ & 55 & $16: 2: 1$ \\
\hline $16^{\mathrm{h}}$ & DIPEA & $\mathrm{CH}_{2} \mathrm{Cl}_{2}$ & $15 \mathrm{~min}$ & 98 & $15: 2: 1$ \\
\hline
\end{tabular}

a Reaction conditions: 1a $(0.25 \mathrm{mmol}), 2$ (0.15 mmol), cat. (10 mol\%), solvent $(2.5 \mathrm{~mL}, 0.1 \mathrm{M})$.

b Yield of 3a after flash chromatography.

c The diastereomeric ratio was determined by ${ }^{1} \mathrm{H}$ NMR.

d HMTA = hexamethylenetetramine.

e The reaction was carried out at $0{ }^{\circ} \mathrm{C}$.

$\mathrm{f}$ The reaction was carried out with DIPEA $(30 \mathrm{~mol} \%)$ at $0{ }^{\circ} \mathrm{C}$.

9 The reaction was carried out with DIPEA (1 mol\%).

h The reaction was carried out with DIPEA (5 mol\%).

lent diastereomeric ratios were achieved by varying the alkyl substituent at C-3 (3o-q). It was observed that an increase in the steric hindrance at C-3 resulted in a lower yield (3n-p).

Furthermore, an enantioselective sulfa-Michael/aldol domino sequence was investigated, catalyzed by different chiral bifunctional organocatalysts such as squaramides ${ }^{12}$ and thioureas. ${ }^{13}$ Initially different squaramide and thiourea catalysts (Table 2, I-IV) were tested to provide the product 3a. In all approaches good to excellent yields could be achieved with good diastereomeric ratios, but the best enantioselectivity of only $19 \%$ ee was obtained with catalyst I. The reaction was then conducted in different solvents and at low temperature employing catalyst I, but so far the ee value could not be increased beyond $23 \%$ (entry 6 ). 


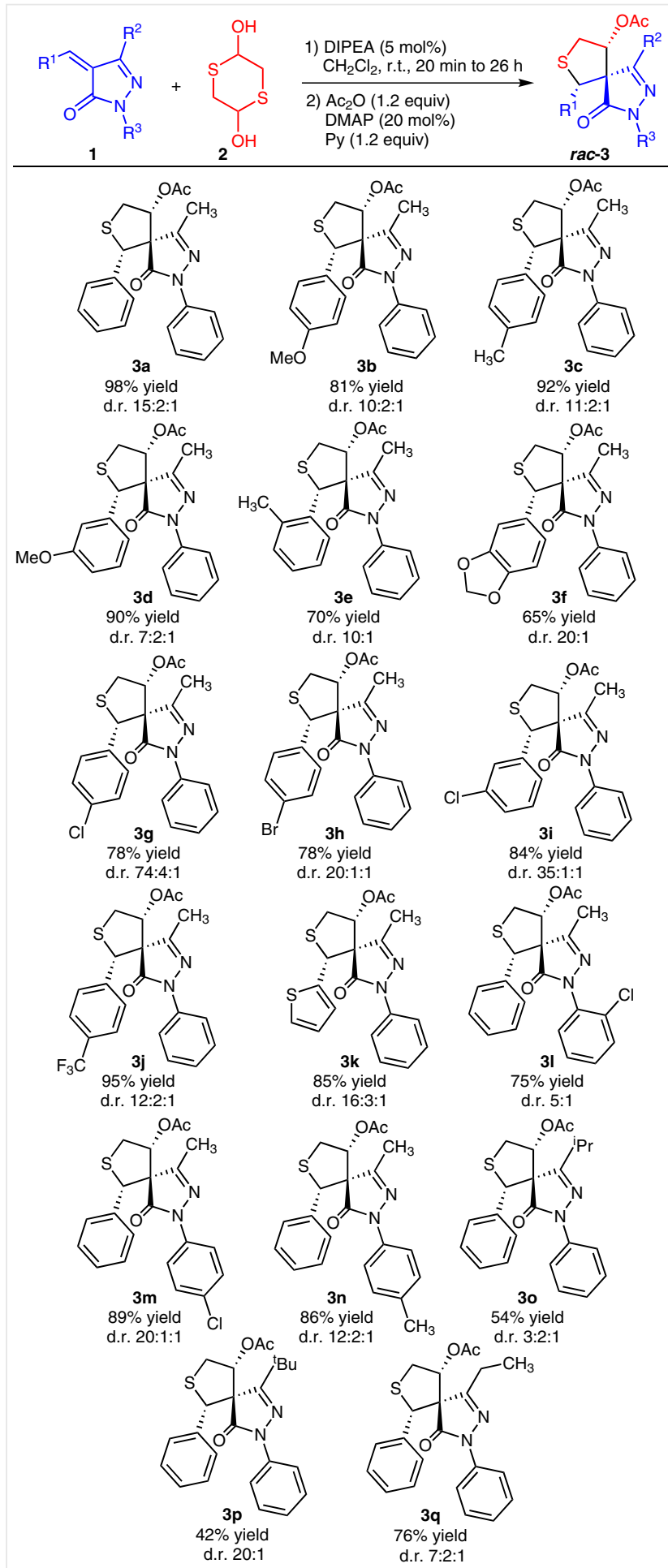

Scheme 2 Substrate scope of the sulfa-Michael/aldol domino reaction
Table 2 Test of an Enantioselective Sulfa-Michael/Aldol Domino Reaction $^{\mathrm{a}}$<smiles>CC1=NN(c2ccccc2)C(=O)C1=Cc1ccccc1</smiles>

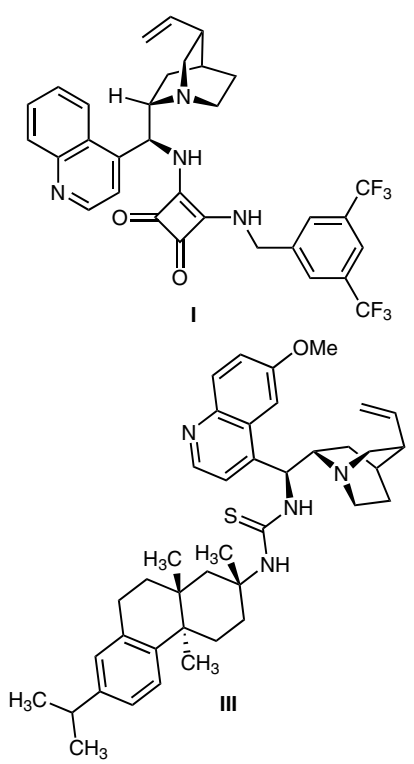<smiles>C=CC1CC2CCN1C2(C)C(Nc1c(Nc2cc(C(F)(F)F)cc(C(F)(F)F)c2)c(=O)c1=O)c1ccnc2ccc(OC)cc12</smiles><smiles>C=CC1CC2CCN1C2C(NC(=S)Nc1cc(C(F)(F)F)cc(C(F)(F)F)c1)c1ccnc2ccccc12</smiles>

\begin{tabular}{llllllr}
\hline Entry & Catalyst & Solvent & $\begin{array}{l}\text { Reaction } \\
\text { time }\end{array}$ & $\begin{array}{l}\text { Yield } \\
(\%)^{\mathrm{b}}\end{array}$ & $\begin{array}{l}\text { ee } \\
(\%)^{\mathrm{c}}\end{array}$ & d.r. $^{\text {d }}$ \\
\hline 1 & I & $\mathrm{CH}_{2} \mathrm{Cl}_{2}$ & $1 \mathrm{~h}$ & 98 & 19 & $10: 2: 1$ \\
2 & II & $\mathrm{CH}_{2} \mathrm{Cl}_{2}$ & $2.5 \mathrm{~h}$ & 88 & 4 & $12: 2: 1$ \\
3 & III & $\mathrm{CH}_{2} \mathrm{Cl}_{2}$ & $5.5 \mathrm{~h}$ & 98 & 18 & $8: 2: 1$ \\
4 & IV & $\mathrm{CH}_{2} \mathrm{Cl}_{2}$ & $5.5 \mathrm{~h}$ & 98 & 14 & $10: 2: 1$ \\
5 & I & toluene & $3 \mathrm{~d}$ & 97 & 17 & $6: 1: 1$ \\
6 & I & THF & $24 \mathrm{~h}$ & 66 & 23 & $12: 3: 1$ \\
7 e & I & THF & $2 \mathrm{~d}$ & 45 & 21 & $13: 3: 1$
\end{tabular}

a Reaction conditions: 1a $(0.25 \mathrm{mmol}), 2$ ( $0.15 \mathrm{mmol})$, cat. ( $5 \mathrm{~mol} \%)$, solvent $(2.5 \mathrm{~mL}, 0.1 \mathrm{M})$.

b Yield of $3 a$ after flash chromatography.

c The enantiomeric excess was determined by HPLC on a chiral stationary phase.

${ }^{\mathrm{d}}$ The diastereomeric ratio was determined by ${ }^{1} \mathrm{H}$ NMR.

e The reaction was carried out at $-65^{\circ} \mathrm{C}$.

The relative cis-configuration of the spiro[pyrazolone4,3'-tetrahydrothiophenes] was determined by X-ray crystal structure analysis of compound $\mathbf{3 i}$ (Figure 2). ${ }^{14}$

In conclusion, we have developed a diastereoselective sulfa-Michael/aldol domino reaction of arylidenepyrazolones with 1,4-dithiane-2,5-diol to afford spiro[pyrazolone4,3'-tetrahydrothiophenes] in moderate to excellent yields and diastereomeric ratios. An attempt to develop an enantioselective variant of this protocol has also been carried out, but so far only $23 \%$ ee could be reached. 

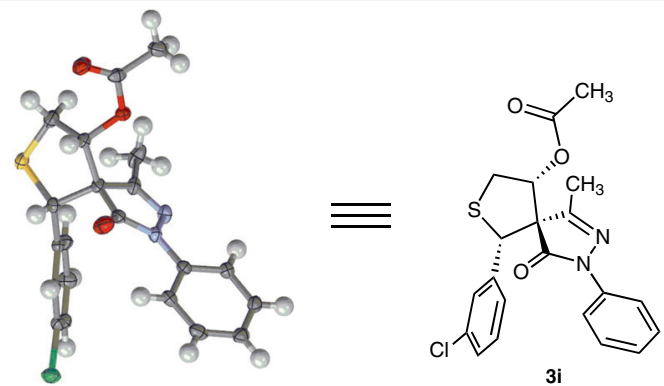

3i

Figure 2 X-ray crystal structure of spiro[pyrazolone-4,3'-tetrahydrothiophene] 3i. Interestingly, one enantiomer of $\mathbf{3 i}$ was obtained after crystallization from $n$-hexane-ethyl acetate (1:1).

Unless otherwise noted, all commercially available chemicals were used without purification. All solvents were distilled and purified according to standard procedures. Analytical TLC was performed using SIL G-25 UV ${ }_{254}$ from Macherey \& Nagel (particle size 0.040-0.063 nm; 230-240 mesh flash) and visualized with UV radiation at $254 \mathrm{~nm} .{ }^{1} \mathrm{H}$, ${ }^{13} \mathrm{C}$, and ${ }^{19} \mathrm{~F}$ NMR spectra were recorded at ambient temperature on a Varian Innova 400 or Innova 600 instrument. Chemical shifts of the major diastereomer for the ${ }^{1} \mathrm{H}$ NMR and ${ }^{13} \mathrm{C}$ NMR spectra are reported in ppm with coupling constants given in $\mathrm{Hz}$. Melting points were measured on a LLG MPM-H2 melting point instrument. Mass spectra were acquired on a Finnigan SSQ7000 (EI, $70 \mathrm{eV}$ ) spectrometer and on a ThermoFinnigan LCQ Deca XP plus (ESI) spectrometer, and high resolution ESI spectra were obtained on a ThermoFisher Scientific LTQ Orbitrap XL. Analytical HPLC was performed on a Agilent 1260 instrument by using chiral stationary phases (Daicel Chiralpak IA column). The alkylidenepyrazolones $\mathbf{1}$ were prepared according to known procedures. ${ }^{15}$

\section{Compounds rac-3a-q; General Procedure}

1,4-Dithiane-2,5-diol (2; $0.15 \mathrm{mmol})$ and DIPEA (5 mol\%) were added to a solution of $1(0.25 \mathrm{mmol})$ in $\mathrm{CH}_{2} \mathrm{Cl}_{2}(2.5 \mathrm{~mL})$. The reaction mixture was stirred at r.t. until $\mathbf{1}$ was completely consumed as indicated by TLC. The solution of the crude product was cooled to $0{ }^{\circ} \mathrm{C}$ and treated with $\mathrm{Ac}_{2} \mathrm{O}(0.30 \mathrm{mmol})$, DMAP $(0.05 \mathrm{mmol})$, and pyridine $(0.30 \mathrm{mmol})$. The crude product was then subjected to flash chromatography (silica gel, $n$-pentane- $\mathrm{Et}_{2} \mathrm{O}, 20: 1$ to $6: 1$ ); this afforded the spiro product 3.

\section{1-Methyl-4-oxo-3,6-diphenyl-7-thia-2,3-diazaspiro[4.4]non-1-en- 9-yl Acetate (3a)}

Yield: $93 \mathrm{mg}$ (98\%); colorless solid; $\mathrm{mp} 78-80{ }^{\circ} \mathrm{C} ; R_{f}=0.60$ ( $n$-pentane- $\left.\mathrm{Et}_{2} \mathrm{O}, 1: 1\right)$.

IR (ATR): 1750, 1709, 1595, 1495, 1452, 1401, 1364, 1323, 1292, 1216 , $1148,1053,918,839,798,758,696 \mathrm{~cm}^{-1}$.

${ }^{1} \mathrm{H}$ NMR $\left(600 \mathrm{MHz}, \mathrm{CDCl}_{3}\right): \delta=7.70(\mathrm{~m}, 2 \mathrm{H}, \mathrm{Ar}-\mathrm{H}), 7.39-7.33(\mathrm{~m}, 4 \mathrm{H}$, Ar-H), 7.25-7.15 (m, $4 \mathrm{H}, \mathrm{Ar}-\mathrm{H}), 5.75(\mathrm{t}, J=8.5 \mathrm{~Hz}, 1 \mathrm{H}, \mathrm{CHOAc}), 5.14$ (s, $1 \mathrm{H}, \mathrm{CHAr}$ ), 3.81 (dd, $J=11.1,8.3 \mathrm{~Hz}, 1 \mathrm{H}, \mathrm{SCHH}), 3.19$ (dd, $J=11.1$, $8.8 \mathrm{~Hz}, 1 \mathrm{H}, \mathrm{SCHH}), 2.38\left(\mathrm{~s}, 3 \mathrm{H}, \mathrm{CH}_{3}\right), 1.98\left(\mathrm{~s}, 3 \mathrm{H}, \mathrm{CH}_{3}\right)$.

${ }^{13} \mathrm{C}$ NMR $\left(151 \mathrm{MHz}, \mathrm{CDCl}_{3}\right): \delta=170.9(\mathrm{C}=\mathrm{O}), 169.3(\mathrm{C}=0), 157.5\left(\mathrm{C}_{\mathrm{q}}\right)$, 137.4 $\left(\mathrm{C}_{\mathrm{q}}\right), 133.1\left(\mathrm{C}_{\mathrm{q}}\right), 128.9$ (2 C, Ar-C), $128.8(\mathrm{Ar}-\mathrm{C}), 128.6$ (2 C, $\left.\mathrm{Ar}-\mathrm{C}\right)$, 127.2 (2 C, Ar-C), 125.5 (Ar-C), 119.2 (2 C, Ar-C), 79.3 (CHOAC), 69.2 $\left(\mathrm{C}_{\mathrm{q}}\right), 54.3(\mathrm{CHAr}), 33.0\left(\mathrm{SCH}_{2}\right), 20.6\left(\mathrm{CH}_{3}\right), 17.8\left(\mathrm{CH}_{3}\right)$.
MS (EI, $70 \mathrm{eV}): m / z(\%)=381.3(7)[\mathrm{M}+\mathrm{H}]^{+}, 380.1(33)[\mathrm{M}]^{+}, 320.1(55)$ $[\mathrm{M}-\mathrm{OAc}]^{+}, 263.1(100)\left[\mathrm{M}-\mathrm{C}_{4} \mathrm{H}_{5} \mathrm{O}_{2} \mathrm{~S}\right]^{+}$.

HRMS (ESI): $m / z[M+H]^{+}$calcd for $\mathrm{C}_{21} \mathrm{H}_{21} \mathrm{~N}_{2} \mathrm{O}_{3} \mathrm{~S}$ : 381.1267; found: 381.1268 .

\section{6-(4-Methoxyphenyl)-1-methyl-4-oxo-3-phenyl-7-thia-2,3-di-} azaspiro[4.4]non-1-en-9-yl Acetate (3b)

Yield: $83 \mathrm{mg}$ (81\%); yellow solid; $\mathrm{mp} 38-40{ }^{\circ} \mathrm{C} ; R_{f}=0.47$ ( $n$-pentane$\left.\mathrm{Et}_{2} \mathrm{O}, 1: 1\right)$.

IR (ATR): 2944, 1719, 1596, 1493, 1363, 1216, 1044, 918, 829, 753, $692 \mathrm{~cm}^{-1}$.

${ }^{1} \mathrm{H}$ NMR $\left(600 \mathrm{MHz}, \mathrm{CDCl}_{3}\right): \delta=7.72(\mathrm{~d}, J=8.6 \mathrm{~Hz}, 2 \mathrm{H}, \mathrm{Ar}-\mathrm{H}), 7.36(\mathrm{~m}$, $2 \mathrm{H}, \mathrm{Ar}-\mathrm{H}), 7.29-7.23(\mathrm{~m}, 2 \mathrm{H}, \mathrm{Ar}-\mathrm{H}), 7.17(\mathrm{~m}, 1 \mathrm{H}, \mathrm{Ar}-\mathrm{H}), 6.74(\mathrm{t}, J=7.7$ $\mathrm{Hz}, 2 \mathrm{H}, \mathrm{Ar}-\mathrm{H}$ ), 5.72 (t, J = 8.5 Hz, $1 \mathrm{H}, \mathrm{CHOAc}), 5.09$ (s, $1 \mathrm{H}, \mathrm{CHAr}$ ), 3.80 (dd, $J=11.0,8.5 \mathrm{~Hz}, 1 \mathrm{H}, \mathrm{SCHH}), 3.72\left(\mathrm{~s}, 3 \mathrm{H}, \mathrm{OCH}_{3}\right), 3.17(\mathrm{~m}, 1 \mathrm{H}$, $\mathrm{SCHH}), 2.40\left(\mathrm{~s}, 3 \mathrm{H}, \mathrm{CH}_{3}\right), 1.97\left(\mathrm{~s}, 3 \mathrm{H}, \mathrm{CH}_{3}\right)$.

${ }^{13} \mathrm{C}$ NMR $\left(151 \mathrm{MHz}, \mathrm{CDCl}_{3}\right): \delta=171.0(\mathrm{C}=0), 169.3(\mathrm{C}=0), 159.7\left(\mathrm{C}_{\mathrm{q}}\right)$, $157.6\left(C_{q}\right), 137.4\left(C_{q}\right), 129.6\left(C_{q}\right), 128.8(2 \mathrm{C}, \mathrm{Ar}-\mathrm{C}), 128.4(2 \mathrm{C}, \mathrm{Ar}-\mathrm{C})$, 125.4 (Ar-C), 119.1 (2 C, Ar-C), 113.9 (2 C, Ar-C), 79.2 (CHOAC), 69.2 $\left(\mathrm{C}_{\mathrm{q}}\right), 55.2\left(\mathrm{OCH}_{3}\right), 53.9(\mathrm{CHAr}), 33.0\left(\mathrm{SCH}_{2}\right), 20.6\left(\mathrm{CH}_{3}\right), 17.8\left(\mathrm{CH}_{3}\right)$.

MS (EI, $70 \mathrm{eV}): m / z(\%)=411.1(16)[\mathrm{M}+\mathrm{H}]^{+}, 410.1(62)[\mathrm{M}]^{+}, 350.1$ (50) $[\mathrm{M}-\mathrm{HOAc}]^{+}, 293.1(100)\left[\mathrm{M}-\mathrm{C}_{4} \mathrm{H}_{5} \mathrm{O}_{2} \mathrm{~S}\right]^{+}$.

HRMS (ESI): $m / z$ [M + Na] $]^{+}$calcd for $\mathrm{C}_{22} \mathrm{H}_{22} \mathrm{~N}_{2} \mathrm{O}_{4} \mathrm{SNa}$ : 433.1193; found: 433.1192 .

1-Methyl-4-oxo-3-phenyl-6-p-tolyl-7-thia-2,3-diazaspiro[4.4]non-1-en-9-yl Acetate (3c)

Yield: $91 \mathrm{mg}$ (92\%); colorless solid; $\mathrm{mp} 35-37{ }^{\circ} \mathrm{C} ; R_{f}=0.68$ (n-pentane- $\left.\mathrm{Et}_{2} \mathrm{O}, 1: 1\right)$.

IR (ATR): 2951, 1750, 1709, 1596, 1497, 1434, 1402, 1364, 1291, 1216, 1146, 1052, 920, 821, 798, 757, 727, $689 \mathrm{~cm}^{-1}$.

${ }^{1} \mathrm{H}$ NMR $\left(600 \mathrm{MHz}, \mathrm{CDCl}_{3}\right): \delta=7.72(\mathrm{~m}, 2 \mathrm{H}, \mathrm{Ar}-\mathrm{H}), 7.36(\mathrm{~m}, 2 \mathrm{H}, \mathrm{Ar}-\mathrm{H})$, $7.22(\mathrm{~d}, J=8.1 \mathrm{~Hz}, 2 \mathrm{H}, \mathrm{Ar}-\mathrm{H}), 7.18(\mathrm{~m}, 1 \mathrm{H}, \mathrm{Ar}-\mathrm{H}), 7.01$ (d, $J=7.9 \mathrm{~Hz}, 2$ $\mathrm{H}, \mathrm{Ar}-\mathrm{H}), 5.73(\mathrm{t}, J=8.5 \mathrm{~Hz}, 1 \mathrm{H}, \mathrm{CHOAc}), 5.11(\mathrm{~s}, 1 \mathrm{H}, \mathrm{CHAr}), 3.80$ (dd, $J=11.1,8.3 \mathrm{~Hz}, 1 \mathrm{H}, \mathrm{SCH}), 3.17(\mathrm{dd}, J=11.1,8.8 \mathrm{~Hz}, 1 \mathrm{H}, \mathrm{SCHH}), 2.39$ (s, $\left.3 \mathrm{H}, \mathrm{CH}_{3}\right), 2.25\left(\mathrm{~s}, 3 \mathrm{H}, \mathrm{CH}_{3}\right), 1.98\left(\mathrm{~s}, 3 \mathrm{H}, \mathrm{CH}_{3}\right)$.

${ }^{13} \mathrm{C}$ NMR (151 MHz, $\left.\mathrm{CDCl}_{3}\right): \delta=171.0(\mathrm{C}=0), 169.3(\mathrm{C}=0), 157.6\left(\mathrm{C}_{\mathrm{q}}\right)$, $138.6\left(C_{q}\right), 137.5\left(C_{q}\right), 129.9\left(C_{q}\right), 129.3(2 \mathrm{C}, \operatorname{Ar}-\mathrm{C}), 128.8$ (2 C, Ar-C), 127.0 (2 C, Ar-C), 125.4 (Ar-C), 119.1 (2 C, Ar-C), 79.4 (CHOAC), 69.2 $\left(\mathrm{C}_{\mathrm{q}}\right), 54.1$ ( $\left.\mathrm{CHAr}\right), 33.0\left(\mathrm{SCH}_{2}\right), 21.1\left(\mathrm{CH}_{3}\right), 20.6\left(\mathrm{CH}_{3}\right), 17.8\left(\mathrm{CH}_{3}\right)$.

MS (EI, $70 \mathrm{eV}): m / z(\%)=395.2(11)[\mathrm{M}+\mathrm{H}]^{+}, 394.2(31)[\mathrm{M}]^{+}, 334.1$ (64) $[\mathrm{M}-\mathrm{HOAc}]^{+}, 277.1(100)\left[\mathrm{M}-\mathrm{C}_{4} \mathrm{H}_{5} \mathrm{O}_{2} \mathrm{~S}\right]^{+}$.

HRMS (ESI): $m / z$ [M + Na $]^{+}$calcd for $\mathrm{C}_{22} \mathrm{H}_{22} \mathrm{~N}_{2} \mathrm{O}_{3} \mathrm{SNa}$ : 417.1243; found: 417.1240.

6-(3-Methoxyphenyl)-1-methyl-4-oxo-3-phenyl-7-thia-2,3-diazaspiro[4.4]non-1-en-9-yl Acetate (3d)

Yield: $92 \mathrm{mg}$ (90\%); brown oil; $R_{f}=0.55$ (n-pentane- $\mathrm{Et}_{2} \mathrm{O}, 1: 1$ ). IR (ATR): 2951, 1751, 1709, 1595, 1493, 1458, 1433, 1402, 1366, 1270, $1216,1153,1045,923,872,756,713,692 \mathrm{~cm}^{-1}$.

${ }^{1} \mathrm{H}$ NMR $\left(400 \mathrm{MHz}, \mathrm{CDCl}_{3}\right): \delta=7.72(\mathrm{~d}, J=7.8 \mathrm{~Hz}, 2 \mathrm{H}, \mathrm{Ar}-\mathrm{H}), 7.34(\mathrm{t}$, $J=7.9 \mathrm{~Hz}, 2 \mathrm{H}, \mathrm{Ar}-\mathrm{H}), 7.19-7.08(\mathrm{~m}, 2 \mathrm{H}, \mathrm{Ar}-\mathrm{H}), 6.93(\mathrm{~d}, J=7.5 \mathrm{~Hz}, 1 \mathrm{H}$, Ar-H), $6.84(\mathrm{~s}, 1 \mathrm{H}, \mathrm{Ar}-\mathrm{H}), 6.75(\mathrm{~d}, J=8.4 \mathrm{~Hz}, 1 \mathrm{H}, \mathrm{Ar}-\mathrm{H}), 5.72(\mathrm{t}, J=8.6$ $\mathrm{Hz}, 1 \mathrm{H}, \mathrm{CHOAc}), 5.09$ (s, $1 \mathrm{H}, \mathrm{CHAr}), 3.78$ (dd, $J=11.0,8.3 \mathrm{~Hz}, 1 \mathrm{H}$, $\mathrm{SCHH}), 3.56\left(\mathrm{~s}, 3 \mathrm{H} \mathrm{OCH}_{3}\right), 3.16(\mathrm{dd}, J=10.9,9.0 \mathrm{~Hz}, 1 \mathrm{H}, \mathrm{SCHH}), 2.36$ $\left(\mathrm{s}, 3 \mathrm{H}, \mathrm{CH}_{3}\right), 1.96\left(\mathrm{~s}, 3 \mathrm{H}, \mathrm{CH}_{3}\right)$. 
${ }^{13} \mathrm{C}$ NMR $\left(101 \mathrm{MHz}, \mathrm{CDCl}_{3}\right): \delta=171.0(\mathrm{C}=0), 169.2(\mathrm{C}=0), 159.6\left(\mathrm{C}_{\mathrm{q}}\right)$, $157.5\left(C_{q}\right), 137.4\left(C_{q}\right), 134.6\left(C_{q}\right), 129.6(\operatorname{Ar}-C), 128.8(2 \mathrm{C}, \operatorname{Ar}-\mathrm{C}), 125.4$ (Ar-C), 119.3 (Ar-C), 119.0 (2 C, Ar-C), 114.8 (Ar-C), 112.2 (Ar-C), 79.3 (CHOAc), $69.1\left(\mathrm{C}_{\mathrm{q}}\right), 55.0\left(\mathrm{OCH}_{3}\right), 54.1(\mathrm{CHAr}), 33.0\left(\mathrm{SCH}_{2}\right), 20.6\left(\mathrm{CH}_{3}\right)$, $17.8\left(\mathrm{CH}_{3}\right)$.

MS (EI, $70 \mathrm{eV}): m / z(\%)=411.0(9)[\mathrm{M}+\mathrm{H}]^{+}, 410.0(21)[\mathrm{M}]^{+}, 350.0(38)$ $[\mathrm{M}-\mathrm{HOAc}]^{+}, 292.9(100)\left[\mathrm{M}-\mathrm{C}_{4} \mathrm{H}_{5} \mathrm{O}_{2} \mathrm{~S}\right]^{+}$.

HRMS (ESI): $m / z$ [M + Na] $]^{+}$calcd for $\mathrm{C}_{22} \mathrm{H}_{22} \mathrm{~N}_{2} \mathrm{O}_{4} \mathrm{SNa}$ : 433.1193; found: 433.1193.

\section{1-Methyl-4-oxo-3-phenyl-6-o-tolyl-7-thia-2,3-diazaspiro[4.4]non- 1-en-9-yl Acetate (3e)}

Yield: $69 \mathrm{mg}$ (70\%); colorless solid; $\mathrm{mp} 127-129{ }^{\circ} \mathrm{C} ; R_{f}=0.59$ (n-pentane-Et $\left.{ }_{2} \mathrm{O}, 1: 1\right)$.

IR (ATR): 2958, 2312, 2093, 1724, 1476, 1361, 1212, 1025, $737 \mathrm{~cm}^{-1}$. ${ }^{1} \mathrm{H}$ NMR $\left(400 \mathrm{MHz}, \mathrm{CDCl}_{3}\right): \delta=7.75-7.65(\mathrm{~m}, 3 \mathrm{H}, \mathrm{Ar}-\mathrm{H}), 7.35-7.28$ (m, $2 \mathrm{H}, \mathrm{Ar}-\mathrm{H}), 7.17-7.09(\mathrm{~m}, 3 \mathrm{H}, \mathrm{Ar}-\mathrm{H}), 7.02(\mathrm{~m}, 1 \mathrm{H}, \mathrm{Ar}-\mathrm{H}), 5.73(\mathrm{t}$, $J=8.3 \mathrm{~Hz}, 1 \mathrm{H}, \mathrm{CHOAc}), 5.41(\mathrm{~s}, 1 \mathrm{H}, \mathrm{CHAr}), 3.82(\mathrm{dd}, J=11.2,8.1 \mathrm{~Hz}, 1$ $\mathrm{H}, \mathrm{SCHH}$ ), 3.21 (dd, $J=11.2,8.5 \mathrm{~Hz}, 1 \mathrm{H}, \mathrm{SCHH}), 2.53\left(\mathrm{~s}, 3 \mathrm{H}, \mathrm{CH}_{3}\right), 2.26$ (s, $\left.3 \mathrm{H}, \mathrm{CH}_{3}\right), 1.98\left(\mathrm{~s}, 3 \mathrm{H}, \mathrm{CH}_{3}\right)$.

${ }^{13} \mathrm{C}$ NMR $\left(101 \mathrm{MHz}, \mathrm{CDCl}_{3}\right): \delta=170.9(\mathrm{C}=0), 169.2(\mathrm{C}=0), 157.8\left(\mathrm{C}_{\mathrm{q}}\right)$, 137.5 $\left(\mathrm{C}_{\mathrm{q}}\right), 137.3\left(\mathrm{C}_{\mathrm{q}}\right), 131.6\left(\mathrm{C}_{\mathrm{q}}\right), 131.0(\mathrm{Ar}-\mathrm{C}), 128.8(2 \mathrm{C}, \mathrm{Ar}-\mathrm{C}), 128.5$ (Ar-C), 128.1 (Ar-C), 125.9 (Ar-C), 125.3 (Ar-C), 118.8 (2 C, Ar-C), 79.6 (CHOAC), $68.2\left(\mathrm{C}_{\mathrm{q}}\right), 50.3(\mathrm{CHAr}), 33.4\left(\mathrm{SCH}_{2}\right), 20.7\left(\mathrm{CH}_{3}\right), 19.6\left(\mathrm{CH}_{3}\right)$, $18.6\left(\mathrm{CH}_{3}\right)$

MS (EI, $70 \mathrm{eV}): m / z(\%)=395.2(1)[\mathrm{M}+\mathrm{H}]^{+}, 335.0(1)[\mathrm{M}-\mathrm{OAc}]^{+}$.

HRMS (ESI): $m / z$ [M $+\mathrm{Na}]^{+}$calcd for $\mathrm{C}_{22} \mathrm{H}_{22} \mathrm{~N}_{2} \mathrm{O}_{3} \mathrm{SNa}$ : 417.1243; found: 417.1226.

\section{6-(Benzo[d][1,3]dioxol-5-yl)-1-methyl-4-oxo-3-phenyl-7-thia-2,3- diazaspiro[4.4]non-1-en-9-yl Acetate (3f)}

Yield: $69 \mathrm{mg}$ (65\%); orange solid; $\mathrm{mp} 42-44^{\circ} \mathrm{C} ; R_{f}=0.63$ (n-pentane$\left.\mathrm{Et}_{2} \mathrm{O}, 1: 1\right)$.

IR (ATR): 2902, 1717, 1598, 1488, 1362, 1221, 1037, 922, $758 \mathrm{~cm}^{-1}$.

${ }^{1} \mathrm{H} \mathrm{NMR}\left(600 \mathrm{MHz}, \mathrm{CDCl}_{3}\right): \delta=7.73(\mathrm{~m}, 2 \mathrm{H}, \mathrm{Ar}-\mathrm{H}), 7.40-7.34(\mathrm{~m}, 2 \mathrm{H}$, Ar-H), $7.18(\mathrm{~m}, 1 \mathrm{H}, \mathrm{Ar}-\mathrm{H}), 6.91(\mathrm{~d}, J=1.8 \mathrm{~Hz}, 1 \mathrm{H}, \mathrm{Ar}-\mathrm{C}), 6.80$ (dd, $J=$ $8.2,1.9 \mathrm{~Hz}, 1 \mathrm{H}, \mathrm{Ar}-\mathrm{H}), 6.62(\mathrm{~d}, J=8.1 \mathrm{~Hz}, 1 \mathrm{H}, \mathrm{Ar}-\mathrm{H}), 5.90$ (dd, $J=12.0$, $1.5 \mathrm{~Hz}, 2 \mathrm{H},-\mathrm{OCH}_{2} \mathrm{O}-$ ), 5.70 (t, $\left.J=8.6 \mathrm{~Hz}, 1 \mathrm{H}, \mathrm{CHOAc}\right), 5.05(\mathrm{~s}, 1 \mathrm{H}$, CHAr), 3.79 (dd, $J=11.1,8.3 \mathrm{~Hz}, 1 \mathrm{H}, \mathrm{SCHH}), 3.17$ (dd, $J=11.1,8.9 \mathrm{~Hz}$, $1 \mathrm{H}, \mathrm{SCHH}), 2.42\left(\mathrm{~s}, 3 \mathrm{H}, \mathrm{CH}_{3}\right), 1.98\left(\mathrm{~s}, 3 \mathrm{H}, \mathrm{CH}_{3}\right)$.

${ }^{13} \mathrm{C}$ NMR (151 MHz, $\left.\mathrm{CDCl}_{3}\right): \delta=170.8(\mathrm{C}=0), 169.2(\mathrm{C}=0), 157.5\left(\mathrm{C}_{\mathrm{q}}\right)$, $147.9\left(\mathrm{C}_{\mathrm{q}}\right), 147.8\left(\mathrm{C}_{\mathrm{q}}\right), 137.4\left(\mathrm{C}_{\mathrm{q}}\right), 128.9(2 \mathrm{C}, \mathrm{Ar}-\mathrm{C}), 126.6\left(\mathrm{C}_{\mathrm{q}}\right), 125.5$ (Ar-C), 121.0 (Ar-C), 119.2 (2 C, Ar-C), 108.2 (Ar-C), 107.5 (Ar-C), $101.3\left(-\mathrm{OCH}_{2} \mathrm{O}-\right)$, 79.1 (CHOAc), $69.2\left(\mathrm{C}_{\mathrm{q}}\right), 54.2(\mathrm{CHAr}), 33.0\left(\mathrm{SCH}_{2}\right)$, $20.6\left(\mathrm{CH}_{3}\right), 17.9\left(\mathrm{CH}_{3}\right)$.

MS (EI, $70 \mathrm{eV}): m / z(\%)=425.3(2)[\mathrm{M}+\mathrm{H}]^{+}, 424.2(7)[\mathrm{M}]^{+}, 364.2(16)$ $[\mathrm{M}-\mathrm{HOAC}]^{+}, 307.1(100)\left[\mathrm{M}-\mathrm{C}_{4} \mathrm{H}_{5} \mathrm{O}_{2} \mathrm{~S}\right]^{+}$.

HRMS (ESI): $m / z$ [M + Na] $]^{+}$calcd for $\mathrm{C}_{22} \mathrm{H}_{20} \mathrm{~N}_{2} \mathrm{O}_{5} \mathrm{SNa}$ : 447.0985; found: 447.0984 .

\section{6-(4-Chlorophenyl)-1-methyl-4-oxo-3-phenyl-7-thia-2,3-diaza-} spiro[4.4]non-1-en-9-yl Acetate (3g)

Yield: $81 \mathrm{mg}$ (78\%); colorless solid; $\mathrm{mp} 37-39{ }^{\circ} \mathrm{C} ; R_{f}=0.50$ (n-pentane- $\left.\mathrm{Et}_{2} \mathrm{O}, 1: 1\right)$.
IR (ATR): 2939, 2291, 2083, 1715, 1596, 1491, 1361, 1213, 1043, 917 , $828,751,691 \mathrm{~cm}^{-1}$.

${ }^{1} \mathrm{H}$ NMR $\left(600 \mathrm{MHz}, \mathrm{CDCl}_{3}\right): \delta=7.74-7.70(\mathrm{~m}, 2 \mathrm{H}, \mathrm{Ar}-\mathrm{H}), 7.37(\mathrm{~m}, 2 \mathrm{H}$, Ar-H), 7.31-7.27 (m, 2 H, Ar-H), 7.22-7.18 (m, 3 H, Ar-H), 5.73 (t, J = $8.6 \mathrm{~Hz}, 1 \mathrm{H}, \mathrm{CHOAc}), 5.09$ (s, $1 \mathrm{H}, \mathrm{CHAr}$ ), 3.81 (dd, $J=11.1,8.3 \mathrm{~Hz}, 1 \mathrm{H}$, $\mathrm{SCHH}), 3.19$ (dd, $J=11.1,8.9 \mathrm{~Hz}, 1 \mathrm{H}, \mathrm{SCHH}), 2.37$ (s, $\left.3 \mathrm{H}, \mathrm{CH}_{3}\right), 1.98$ (s, $\left.3 \mathrm{H}, \mathrm{CH}_{3}\right)$.

${ }^{13} \mathrm{C}$ NMR $\left(151 \mathrm{MHz}, \mathrm{CDCl}_{3}\right): \delta=170.7(\mathrm{C}=0), 169.2(\mathrm{C}=0), 157.2\left(\mathrm{C}_{\mathrm{q}}\right)$, $137.3\left(\mathrm{C}_{\mathrm{q}}\right), 134.6\left(\mathrm{C}_{\mathrm{q}}\right), 131.8\left(\mathrm{C}_{\mathrm{q}}\right), 128.9(2 \mathrm{C}, \mathrm{Ar}-\mathrm{C}), 128.8(2 \mathrm{C}, \mathrm{Ar}-\mathrm{C})$, 128.6 (2 C, Ar-C), 125.6 (Ar-C), 119.1 (2 C, Ar-C), 79.2 (CHOAC), 69.1 $\left(\mathrm{C}_{\mathrm{q}}\right), 53.5(\mathrm{CHAr}), 33.1\left(\mathrm{SCH}_{2}\right), 20.6\left(\mathrm{CH}_{3}\right), 17.8\left(\mathrm{CH}_{3}\right)$.

MS (EI, $70 \mathrm{eV}): m / z(\%)=416.1(16)\left[\mathrm{M},{ }^{37} \mathrm{Cl}\right]^{+}, 414.1(39)\left[\mathrm{M},{ }^{35} \mathrm{Cl}\right]^{+}$, 356.0 (17) [M - HOAc, $\left.{ }^{37} \mathrm{Cl}\right]^{+}, 354.0(65)\left[\mathrm{M}-\mathrm{HOAc},{ }^{35} \mathrm{Cl}\right]^{+}, 299.1$ (31) $\left[\mathrm{M}-\mathrm{C}_{4} \mathrm{H}_{5} \mathrm{O}_{2} \mathrm{~S},{ }^{37} \mathrm{Cl}\right]^{+}, 297.0(100)\left[\mathrm{M}-\mathrm{C}_{4} \mathrm{H}_{5} \mathrm{O}_{2} \mathrm{~S},{ }^{35} \mathrm{Cl}\right]^{+}$.

HRMS (ESI): $m / z[\mathrm{M}+\mathrm{Na}]^{+}$calcd for $\mathrm{C}_{21} \mathrm{H}_{19} \mathrm{ClN}_{2} \mathrm{O}_{3} \mathrm{SNa}$ : 437.0697; found: 437.0698 .

\section{6-(4-Bromophenyl)-1-methyl-4-oxo-3-phenyl-7-thia-2,3-diaza- spiro[4.4]non-1-en-9-yl Acetate (3h)}

Yield: $90 \mathrm{mg}$ (78\%); colorless solid; $\mathrm{mp} 38-40{ }^{\circ} \mathrm{C} ; R_{f}=0.53$ (n-pentane- $\left.\mathrm{Et}_{2} \mathrm{O}, 1: 1\right)$.

IR (ATR): 1750, 1708, 1595, 1492, 1430, 1398, 1365, 1279, 1214, 1147, $1105,1054,1009,955,920,881,827,793,757,725,691 \mathrm{~cm}^{-1}$.

${ }^{1} \mathrm{H}$ NMR $\left(600 \mathrm{MHz}, \mathrm{CDCl}_{3}\right): \delta=7.74-7.70(\mathrm{~m}, 2 \mathrm{H}, \mathrm{Ar}-\mathrm{H}), 7.40-7.33$ (m, $4 \mathrm{H}, \mathrm{Ar}-\mathrm{H}), 7.24-7.17(\mathrm{~m}, 3 \mathrm{H}, \mathrm{Ar}-\mathrm{H}), 5.73(\mathrm{t}, J=8.6 \mathrm{~Hz}, 1 \mathrm{H}$, CHOAc), 5.07 (s, $1 \mathrm{H}, \mathrm{CHAr}$ ), 3.80 (dd, $J=11.1,8.3 \mathrm{~Hz}, 1 \mathrm{H}, \mathrm{SCHH}), 3.19$ (dd, $J=11.1,8.9 \mathrm{~Hz}, 1 \mathrm{H}, \mathrm{SCHH}), 2.37\left(\mathrm{~s}, 3 \mathrm{H}, \mathrm{CH}_{3}\right), 1.98\left(\mathrm{~s}, 3 \mathrm{H}, \mathrm{CH}_{3}\right.$ ).

${ }^{13} \mathrm{C}$ NMR $\left(151 \mathrm{MHz}, \mathrm{CDCl}_{3}\right): \delta=170.7(\mathrm{C}=0), 169.2(\mathrm{C}=0), 157.1\left(\mathrm{C}_{\mathrm{q}}\right)$, $137.3\left(\mathrm{C}_{\mathrm{q}}\right), 132.3\left(\mathrm{C}_{\mathrm{q}}\right), 131.8$ (2 C, Ar-C), 128.9 (2 C, Ar-C), 128.9 (2 C, Ar-C), 125.6 (Ar-C), $122.8\left(\mathrm{C}_{\mathrm{q}}\right), 119.1$ (2 C, Ar-C), 79.2 (CHOAC), 68.98 $\left(\mathrm{C}_{\mathrm{q}}\right), 53.5(\mathrm{CHAr}), 33.1\left(\mathrm{SCH}_{2}\right), 20.6\left(\mathrm{CH}_{3}\right), 17.8\left(\mathrm{CH}_{3}\right)$.

MS (EI, $70 \mathrm{eV}): m / z(\%)=460.1(34)\left[\mathrm{M},{ }^{81} \mathrm{Br}\right]^{+}, 458.0(33)\left[\mathrm{M},{ }^{79} \mathrm{Br}\right]^{+}$, $400.0(62)\left[\mathrm{M}-\mathrm{OAc},{ }^{81} \mathrm{Br}\right]^{+}, 398.0(60)\left[\mathrm{M}-\mathrm{OAc},{ }^{79} \mathrm{Br}\right]^{+}, 343.1(94)[\mathrm{M}$ $\left.-\mathrm{C}_{4} \mathrm{H}_{6} \mathrm{O}_{2} \mathrm{~S},{ }^{81} \mathrm{Br}\right]^{+}, 341.0(100)\left[\mathrm{M}-\mathrm{C}_{4} \mathrm{H}_{6} \mathrm{O}_{2} \mathrm{~S},{ }^{79} \mathrm{Br}\right]^{+}$.

HRMS (ESI): $m / z[\mathrm{M}+\mathrm{Na}]^{+}$calcd for $\mathrm{C}_{21} \mathrm{H}_{19} \mathrm{~N}_{2} \mathrm{O}_{3} \mathrm{~S}^{79} \mathrm{BrNa}$ : 481.0192; found: 481.0193 .

\section{6-(3-Chlorophenyl)-1-methyl-4-oxo-3-phenyl-7-thia-2,3-diaza-} spiro[4.4]non-1-en-9-yl Acetate (3i)

Yield: $87 \mathrm{mg}$ (84\%); colorless solid; $\mathrm{mp} 119-121{ }^{\circ} \mathrm{C} ; R_{f}=0.59$ ( $n$-pentane- $\left.\mathrm{Et}_{2} \mathrm{O}, 1: 1\right)$.

IR (ATR): 2950, 1728, 1589, 1489, 1361, 1219, 1041, 913, 768, $698 \mathrm{~cm}^{-1}$. ${ }^{1} \mathrm{H}$ NMR $\left(400 \mathrm{MHz}, \mathrm{CDCl}_{3}\right): \delta=7.72-7.66(\mathrm{~m}, 2 \mathrm{H}, \mathrm{Ar}-\mathrm{H}), 7.43(\mathrm{~m}, 1 \mathrm{H}$, Ar-H), 7.39-7.32 (m, $2 \mathrm{H}, \mathrm{Ar}-\mathrm{H}), 7.23-7.10(\mathrm{~m}, 4 \mathrm{H}, \mathrm{Ar}-\mathrm{H}), 5.71(\mathrm{t}, J=$ $8.6 \mathrm{~Hz}, 1 \mathrm{H}, \mathrm{CHOAc}), 5.07$ (s, $1 \mathrm{H}, \mathrm{CHAr}), 3.79$ (dd, $J=11.1,8.3 \mathrm{~Hz}, 1 \mathrm{H}$, $\mathrm{SCHH}), 3.18(\mathrm{dd}, J=11.1,8.9 \mathrm{~Hz}, 1 \mathrm{H}, \mathrm{SCHH}), 2.36\left(\mathrm{~s}, 3 \mathrm{H}, \mathrm{CH}_{3}\right), 1.97(\mathrm{~s}$, $\left.3 \mathrm{H}, \mathrm{CH}_{3}\right)$.

${ }^{13} \mathrm{C}$ NMR $\left(101 \mathrm{MHz}, \mathrm{CDCl}_{3}\right): \delta=170.7(\mathrm{C}=0), 169.2(\mathrm{C}=0), 157.1\left(\mathrm{C}_{\mathrm{q}}\right)$, 137.2 $\left(\mathrm{C}_{\mathrm{q}}\right), 135.4\left(\mathrm{C}_{\mathrm{q}}\right), 134.6\left(\mathrm{C}_{\mathrm{q}}\right), 129.9(\mathrm{Ar}-\mathrm{C}), 129.0(\mathrm{Ar}-\mathrm{C}), 128.9(2 \mathrm{C}$, Ar-C), 127.3 (Ar-C), 125.7 (Ar-C), 125.5 (Ar-C), 119.3 (2 C, Ar-C), 79.1 (CHOAC), $69.0\left(\mathrm{C}_{\mathrm{q}}\right), 53.5(\mathrm{CHAr}), 33.1\left(\mathrm{SCH}_{2}\right), 20.6\left(\mathrm{CH}_{3}\right), 17.7\left(\mathrm{CH}_{3}\right)$.

MS (EI, $70 \mathrm{eV}): m / z(\%)=415.9(14)\left[\mathrm{M},{ }^{37} \mathrm{Cl}\right]^{+}, 414.0(35)\left[\mathrm{M},{ }^{35} \mathrm{Cl}\right]^{+}$, 356.0 (19) $\left[\mathrm{M}-\mathrm{HOAc},{ }^{37} \mathrm{Cl}\right]^{+}, 354.0(48)\left[\mathrm{M}-\mathrm{HOAc},{ }^{35} \mathrm{Cl}\right]^{+}, 298.9(29)$ $\left[\mathrm{M}-\mathrm{C}_{4} \mathrm{H}_{5} \mathrm{O}_{2} \mathrm{~S},{ }^{37} \mathrm{Cl}\right]^{+}, 297.0(100)\left[\mathrm{M}-\mathrm{C}_{4} \mathrm{H}_{5} \mathrm{O}_{2} \mathrm{~S},{ }^{35} \mathrm{Cl}\right]^{+}$.

HRMS (ESI): $m / z[\mathrm{M}+\mathrm{Na}]^{+}$calcd for $\mathrm{C}_{21} \mathrm{H}_{19} \mathrm{~N}_{2} \mathrm{O}_{3} \mathrm{SClNa:}$ 437.0697; found: 437.0696 
1-Methyl-4-oxo-3-phenyl-6-[4-(trifluoromethyl)phenyl]-7-thia2,3-diazaspiro[4.4]non-1-en-9-yl Acetate (3j)

Yield: $107 \mathrm{mg}$ (95\%); colorless solid; mp 40-42 ${ }^{\circ} \mathrm{C} ; R_{f}=0.53$ (n-pentane-Et $\left.{ }_{2} \mathrm{O}, 1: 1\right)$.

IR (ATR): 2101, 1712, 1600, 1497, 1322, 1219, 1119, 1053, 921, 846, $755,688 \mathrm{~cm}^{-1}$.

${ }^{1} \mathrm{H} \mathrm{NMR}\left(600 \mathrm{MHz}, \mathrm{CDCl}_{3}\right): \delta=7.70(\mathrm{~m}, 2 \mathrm{H}, \mathrm{Ar}-\mathrm{H}), 7.48(\mathrm{~m}, 4 \mathrm{H}, \mathrm{Ar}-\mathrm{H})$, $7.37(\mathrm{~m}, 2 \mathrm{H}, \mathrm{Ar}-\mathrm{H}), 7.20$ (t, J = 7.4 Hz, $1 \mathrm{H}, \mathrm{Ar}-\mathrm{H}), 5.75$ (t, $J=8.6 \mathrm{~Hz}, 1$ $\mathrm{H}, \mathrm{CHOAc}), 5.16$ (s, $1 \mathrm{H}, \mathrm{CHAr}), 3.83$ (dd, $J=11.1,8.3 \mathrm{~Hz}, 1 \mathrm{H}, \mathrm{SCHH}$ ), $3.21(\mathrm{dd}, J=11.1,8.9 \mathrm{~Hz}, 1 \mathrm{H}, \mathrm{SCH}), 2.37\left(\mathrm{~s}, 3 \mathrm{H}, \mathrm{CH}_{3}\right), 1.99(\mathrm{~s}, 3 \mathrm{H}$, $\mathrm{CH}_{3}$ ).

${ }^{13} \mathrm{C}$ NMR (151 MHz, $\left.\mathrm{CDCl}_{3}\right): \delta=170.6(\mathrm{C}=0), 169.2(\mathrm{C}=0), 157.0\left(\mathrm{C}_{\mathrm{q}}\right)$, $137.46\left(C_{q}\right), 137.2\left(C_{q}\right), 130.9\left(q, J=32.7 \mathrm{~Hz}, C_{q}\right), 128.9(2 \mathrm{C}, \mathrm{Ar}-\mathrm{C})$, 127.7 (2 C, Ar-C), 125.7 (Ar-C), 125.6 (2 C, Ar-C), 123.7 (q, J = 272.3 Hz, $\left.\mathrm{CF}_{3}\right), 119.1$ (2 C, Ar-C), 79.3 (CHOAc), $69.0\left(\mathrm{C}_{\mathrm{q}}\right), 53.5$ (CHAr), 33.1 $\left(\mathrm{SCH}_{2}\right), 20.6\left(\mathrm{CH}_{3}\right), 17.7\left(\mathrm{CH}_{3}\right)$.

$\left.{ }^{19} \mathrm{~F} \mathrm{NMR} \mathrm{(376} \mathrm{MHz,} \mathrm{CDCl}_{3}\right): \delta=-62.78(\mathrm{~s})$.

MS (EI, $70 \mathrm{eV}): m / z(\%)=449.0(7)[\mathrm{M}+\mathrm{H}]^{+}, 447.9(31)[\mathrm{M}]^{+}, 387.9(33)$ $[\mathrm{M}-\mathrm{HOAc}]^{+}, 330.6(100)\left[\mathrm{M}-\mathrm{C}_{4} \mathrm{H}_{6} \mathrm{O}_{2} \mathrm{~S}\right]^{+}$.

HRMS (ESI): $m / z[\mathrm{M}+\mathrm{Na}]^{+}$calcd for $\mathrm{C}_{22} \mathrm{H}_{19} \mathrm{~N}_{2} \mathrm{O}_{3} \mathrm{~S} \mathrm{~F}_{3} \mathrm{Na}$ : 471.0961; found: 471.0961 .

\section{1-Methyl-4-oxo-3-phenyl-6-(2-thienyl)-7-thia-2,3-diaza-} spiro[4.4]non-1-en-9-yl Acetate (3k)

Yield: $82 \mathrm{mg}$ (85\%); orange solid; $\mathrm{mp} 39-41^{\circ} \mathrm{C} ; R_{f}=0.46$ ( $n$-pentane$\left.\mathrm{Et}_{2} \mathrm{O}, 1: 1\right)$.

IR (ATR): 3073, 2953, 1750, 1708, 1595, 1495, 1431, 1363, 1274, 1215 , $1141,1050,919,849,800,757,697 \mathrm{~cm}^{-1}$.

${ }^{1} \mathrm{H}$ NMR (600 MHz, $\left.\mathrm{CDCl}_{3}\right): \delta$ = 7.81-7.77 (m, $\left.2 \mathrm{H}, \mathrm{Ar}-\mathrm{H}\right), 7.40-7.35$ (m, $2 \mathrm{H}, \mathrm{Ar}-\mathrm{H}), 7.19(\mathrm{~m}, 1 \mathrm{H}, \mathrm{Ar}-\mathrm{H}), 7.16\left(\mathrm{~m}, 1 \mathrm{H}, \mathrm{Ar}-\mathrm{H}_{2 \text {-Thienyl }}\right), 6.97(\mathrm{~m}$, $\left.1 \mathrm{H}, \mathrm{Ar}-\mathrm{H}_{2-\text { Thienyl }}\right), 6.85\left(\mathrm{~m}, 1 \mathrm{H}, \mathrm{Ar}-\mathrm{H}_{2-\text { Thienyl }}\right), 5.69(\mathrm{t}, \mathrm{J}=8.6 \mathrm{~Hz}, 1 \mathrm{H}$, CHOAc), 5.32 (s, $1 \mathrm{H}, \mathrm{CHAr}), 3.80$ (dd, $J=11.1,8.3 \mathrm{~Hz}, 1 \mathrm{H}, \mathrm{SCHH}), 3.21$ (dd, $J=11.0,9.0 \mathrm{~Hz}, 1 \mathrm{H}, \mathrm{SCHH}$ ), $2.47\left(\mathrm{~s}, 3 \mathrm{H}, \mathrm{CH}_{3}\right), 1.98\left(\mathrm{~s}, 3 \mathrm{H}, \mathrm{CH}_{3}\right)$.

${ }^{13} \mathrm{C}$ NMR (151 MHz, $\left.\mathrm{CDCl}_{3}\right): \delta=170.4(\mathrm{C}=\mathrm{O}), 169.2(\mathrm{C}=\mathrm{O}), 157.4\left(\mathrm{C}_{\mathrm{q}}\right)$, $137.5\left(C_{q}\right), 136.6\left(C_{q}\right), 128.9(2 \mathrm{C}, \operatorname{Ar}-\mathrm{C}), 127.1(\mathrm{Ar}-\mathrm{C}), 126.4(\mathrm{Ar}-\mathrm{C})$, 125.8 (Ar-C), 125.5 (Ar-C), 119.1 (2 C, $\mathrm{Ar}-\mathrm{C}), 78.8$ (CHOAC), $69.0\left(\mathrm{C}_{\mathrm{q}}\right)$, 49.3 ( $\mathrm{CHAr}), 33.3\left(\mathrm{SCH}_{2}\right), 20.6\left(\mathrm{CH}_{3}\right), 17.8\left(\mathrm{CH}_{3}\right)$.

MS (EI, $70 \mathrm{eV}): m / z(\%)=387.0(10)[\mathrm{M}+\mathrm{H}]^{+}, 385.9(36)[\mathrm{M}]^{+}, 325.8$ (38) $[\mathrm{M}-\mathrm{HOAc}]^{+}, 268.7$ (100) $\left[\mathrm{M}-\mathrm{C}_{4} \mathrm{H}_{5} \mathrm{O}_{2} \mathrm{~S}\right]^{+}$.

HRMS (ESI): $m / z[\mathrm{M}+\mathrm{Na}]^{+}$calcd for $\mathrm{C}_{19} \mathrm{H}_{18} \mathrm{~N}_{2} \mathrm{O}_{3} \mathrm{~S}_{2} \mathrm{Na}$ : 409.0651; found: 409.0651 .

\section{3-(2-Chlorophenyl)-1-methyl-4-oxo-6-phenyl-7-thia-2,3-diaza- spiro[4.4]non-1-en-9-yl Acetate (31)}

Yield: $78 \mathrm{mg}$ (75\%); colorless solid; $\mathrm{mp} 153-155^{\circ} \mathrm{C} ; R_{f}=0.50$ ( $n$-pentane-Et $\left.{ }_{2} \mathrm{O}, 1: 1\right)$.

IR (ATR): 3016, 2969, 2040, 1740, 1592, 1486, 1443, 1366, 1218, 1052 , $919,763,701 \mathrm{~cm}^{-1}$.

${ }^{1} \mathrm{H} \mathrm{NMR}\left(400 \mathrm{MHz}, \mathrm{CDCl}_{3}\right): \delta=7.43(\mathrm{~m}, 3 \mathrm{H}, \mathrm{Ar}-\mathrm{H}), 7.31-7.25(\mathrm{~m}, 5 \mathrm{H}$, Ar-H), 7.08-7.04 (m, $1 \mathrm{H}, \mathrm{Ar}-\mathrm{H}), 5.76(\mathrm{t}, J=8.6 \mathrm{~Hz}, 1 \mathrm{H}, \mathrm{CHOAc}), 5.14$ (s, $1 \mathrm{H}, \mathrm{CHAr}$ ), 3.78 (dd, $J=11.0,8.3 \mathrm{~Hz}, 1 \mathrm{H}, \mathrm{SCHH}), 3.20$ (dd, $J=11.0$, $8.9 \mathrm{~Hz}, 1 \mathrm{H}, \mathrm{SCHH}), 2.39\left(\mathrm{~s}, 3 \mathrm{H}, \mathrm{CH}_{3}\right), 2.02\left(\mathrm{~s}, 3 \mathrm{H}, \mathrm{CH}_{3}\right)$.

${ }^{13} \mathrm{C}$ NMR (101 MHz, $\left.\mathrm{CDCl}_{3}\right): \delta=169.7(\mathrm{C}=\mathrm{O}), 168.9(\mathrm{C}=\mathrm{O}), 156.5\left(\mathrm{C}_{\mathrm{q}}\right)$, $133.9\left(C_{q}\right), 133.8\left(C_{q}\right), 131.9\left(C_{q}\right), 130.3(\operatorname{Ar}-C), 129.8(\operatorname{Ar}-C), 128.7(4 \mathrm{C}$, Ar-C), 128.5 (2 C, Ar-C), 127.3 ( $\mathrm{Ar}-\mathrm{C}), 75.8$ (CHOAC), $66.5\left(\mathrm{C}_{\mathrm{q}}\right), 49.6$ (CHAr), $30.9\left(\mathrm{SCH}_{2}\right), 20.7\left(\mathrm{CH}_{3}\right), 13.7\left(\mathrm{CH}_{3}\right)$.
MS (EI, $70 \mathrm{eV}): m / z(\%)=356.1(11)\left[\mathrm{M}-\mathrm{HOAc},{ }^{37} \mathrm{Cl}\right]^{+}, 354.1$ (27) $[\mathrm{M}-$ HOAc, $\left.{ }^{35} \mathrm{Cl}\right]^{+}, 299.1(35)\left[\mathrm{M}-\mathrm{C}_{4} \mathrm{H}_{5} \mathrm{O}_{2} \mathrm{~S},{ }^{37} \mathrm{Cl}\right]^{+}, 297.1$ (100) $[\mathrm{M}-$ $\left.\mathrm{C}_{4} \mathrm{H}_{5} \mathrm{O}_{2} \mathrm{~S},{ }^{35} \mathrm{Cl}\right]^{+}$.

HRMS (ESI): $m / z$ [M $+\mathrm{Na}]^{+}$calcd for $\mathrm{C}_{21} \mathrm{H}_{19} \mathrm{~N}_{2} \mathrm{O}_{3} \mathrm{SClNa}$ : 437.0697; found: 437.0685 .

\section{3-(4-Chlorophenyl)-1-methyl-4-oxo-6-phenyl-7-thia-2,3-diaza-} spiro[4.4]non-1-en-9-yl Acetate (3m)

Yield: $92 \mathrm{mg}$ (89\%); yellow oil; $R_{f}=0.61$ ( $n$-pentane- $\mathrm{Et}_{2} \mathrm{O}, 1: 1$ ). IR (ATR): 2951, 1723, 1595, 1490, 1357, 1215, 1143, 1044, 929, 825, $769,697 \mathrm{~cm}^{-1}$.

${ }^{1} \mathrm{H}$ NMR (400 MHz, $\left.\mathrm{CDCl}_{3}\right): \delta=7.70-7.64(\mathrm{~m}, 2 \mathrm{H}, \mathrm{Ar}-\mathrm{H}), 7.29(\mathrm{~m}, 4 \mathrm{H}$, Ar-H), 7.23-7.17 (m, $3 \mathrm{H}, \mathrm{Ar}-\mathrm{H}), 5.72(\mathrm{t}, J=8.5 \mathrm{~Hz}, 1 \mathrm{H}, \mathrm{CHOAc}), 5.10$ (s, $1 \mathrm{H}, \mathrm{CHAr}$ ), 3.79 (dd, $J=11.2,8.3 \mathrm{~Hz}, 1 \mathrm{H}, \mathrm{SCHH}), 3.17$ (dd, $J=11.2$, $8.7 \mathrm{~Hz}, 1 \mathrm{H}, \mathrm{SCHH}), 2.36\left(\mathrm{~s}, 3 \mathrm{H}, \mathrm{CH}_{3}\right), 1.96\left(\mathrm{~s}, 3 \mathrm{H}, \mathrm{CH}_{3}\right)$.

${ }^{13} \mathrm{C}$ NMR (101 MHz, $\left.\mathrm{CDCl}_{3}\right): \delta=170.9(\mathrm{C}=0), 169.2(\mathrm{C}=0), 157.8\left(\mathrm{C}_{\mathrm{q}}\right)$, $135.9\left(C_{q}\right), 132.9\left(C_{q}\right), 130.5\left(C_{q}\right), 128.8$ (3 C, Ar-C), 128.6 (2 C, Ar-C), 127.1 (2 C, Ar-C), 120.1 (2 C, Ar-C), 79.3 (CHOAC), $69.3\left(\mathrm{C}_{\mathrm{q}}\right), 54.5$ (CHAr), $33.1\left(\mathrm{SCH}_{2}\right), 20.6\left(\mathrm{CH}_{3}\right), 17.8\left(\mathrm{CH}_{3}\right)$.

MS (EI, $70 \mathrm{eV}): m / z(\%)=416.0(14)\left[\mathrm{M},{ }^{37} \mathrm{Cl}\right]^{+}, 414.0(36)\left[\mathrm{M},{ }^{35} \mathrm{Cl}\right]^{+}$, 356.0 (18) $\left[\mathrm{M}-\mathrm{HOAc},{ }^{37} \mathrm{Cl}\right]^{+}, 354.0(47)\left[\mathrm{M}-\mathrm{HOAc},{ }^{35} \mathrm{Cl}\right]^{+}, 299.0(31)$ $\left[\mathrm{M}-\mathrm{C}_{4} \mathrm{H}_{5} \mathrm{O}_{2} \mathrm{~S},{ }^{37} \mathrm{Cl}\right]^{+}, 297.0(100)\left[\mathrm{M}-\mathrm{C}_{4} \mathrm{H}_{5} \mathrm{O}_{2} \mathrm{~S},{ }^{35} \mathrm{Cl}\right]^{+}$.

HRMS (ESI): $m / z[\mathrm{M}+\mathrm{Na}]^{+}$calcd for $\mathrm{C}_{21} \mathrm{H}_{19} \mathrm{~N}_{2} \mathrm{O}_{3} \mathrm{SCINa}$ : 437.0697; found: 437.0697 .

\section{1-Methyl-4-oxo-6-phenyl-3-p-tolyl-7-thia-2,3-diaza- spiro[4.4]non-1-en-9-yl Acetate (3n)}

Yield: $85 \mathrm{mg}$ (86\%); brown solid; $\mathrm{mp} 37-39{ }^{\circ} \mathrm{C} ; R_{f}=0.56$ ( $n$-pentane$\left.\mathrm{Et}_{2} \mathrm{O}, 1: 1\right)$.

IR (ATR): 3479, 2940, 2655, 2333, 2099, 1719, 1613, 1510, 1362, 1215 , $1044,919,814,698 \mathrm{~cm}^{-1}$.

${ }^{1} \mathrm{H}$ NMR $\left(600 \mathrm{MHz}, \mathrm{CDCl}_{3}\right): \delta=7.59-7.54(\mathrm{~m}, 2 \mathrm{H}, \mathrm{Ar}-\mathrm{H}), 7.36-7.33$ ( $\mathrm{m}, 2 \mathrm{H}, \mathrm{Ar}-\mathrm{H}), 7.24-7.20$ (m, $3 \mathrm{H}, \mathrm{Ar}-\mathrm{H}), 7.17-7.14(\mathrm{~m}, 2 \mathrm{H}, \mathrm{Ar}-\mathrm{H})$, $5.74(\mathrm{t}, J=8.5 \mathrm{~Hz}, 1 \mathrm{H}, \mathrm{CHOAc}), 5.13(\mathrm{~s}, 1 \mathrm{H}, \mathrm{CHAr}), 3.81$ (dd, $J=11.1$, $8.3 \mathrm{~Hz}, 1 \mathrm{H}, \mathrm{SCHH}), 3.18$ (dd, $J=11.1,8.8 \mathrm{~Hz}, 1 \mathrm{H}, \mathrm{SCHH}), 2.37$ (s, $3 \mathrm{H}$, $\left.\mathrm{CH}_{3}\right), 2.33\left(\mathrm{~s}, 3 \mathrm{H}, \mathrm{ArCH}_{3}\right), 1.98\left(\mathrm{~s}, 3 \mathrm{H}, \mathrm{CH}_{3}\right)$.

${ }^{13} \mathrm{C}$ NMR (151 MHz, $\left.\mathrm{CDCl}_{3}\right): \delta=170.7(\mathrm{C}=0), 169.3(\mathrm{C}=0), 157.3\left(\mathrm{C}_{\mathrm{q}}\right)$, $135.3\left(C_{q}\right), 135.0\left(C_{q}\right), 133.1\left(C_{q}\right), 129.3(2 \mathrm{C}, \operatorname{Ar}-C), 128.7(\operatorname{Ar}-C), 128.6$ (2 C, Ar-C), 127.2 (2 C, Ar-C), 119.2 (2 C, Ar-C), 79.3 (CHOAC), $69.1\left(\mathrm{C}_{\mathrm{q}}\right)$, 54.2 ( $\mathrm{CHAr}), 33.0\left(\mathrm{SCH}_{2}\right), 21.0\left(\mathrm{CH}_{3}\right), 20.6\left(\mathrm{CH}_{3}\right), 17.8\left(\mathrm{CH}_{3}\right)$.

MS (EI, $70 \mathrm{eV}): m / z(\%)=395.2(10)[\mathrm{M}+\mathrm{H}]^{+}, 394.2(26)[\mathrm{M}]^{+}, 334.1$ (21) $[\mathrm{M}-\mathrm{HOAc}]^{+}, 277.1(100)\left[\mathrm{M}-\mathrm{C}_{4} \mathrm{H}_{5} \mathrm{O}_{2} \mathrm{~S}\right]^{+}$.

HRMS (ESI): $m / z$ [M + Na $]^{+}$calcd for $\mathrm{C}_{22} \mathrm{H}_{22} \mathrm{~N}_{2} \mathrm{O}_{3} \mathrm{SNa}$ : 395.1424; found: 395.1423.

\section{1-Isopropyl-4-oxo-3,6-diphenyl-7-thia-2,3-diazaspiro[4.4]non-1-} en-9-yl Acetate (3o)

Yield: $55 \mathrm{mg}$ (54\%); yellow solid; $\mathrm{mp} 101-103{ }^{\circ} \mathrm{C} ; R_{f}=0.63$ ( $n$-pentane-Et $\left.{ }_{2} \mathrm{O}, 1: 1\right)$.

IR (ATR): 2973, 1749, 1710, 1596, 1495, 1452, 1355, 1217, 1148, 1051, $979,921,883,753,693 \mathrm{~cm}^{-1}$.

${ }^{1} \mathrm{H}$ NMR $\left(600 \mathrm{MHz}, \mathrm{CDCl}_{3}\right): \delta=7.79\left(\mathrm{~m}, 2 \mathrm{H}, \mathrm{Ar}-\mathrm{H}_{\text {Diast }}\right), 7.52(\mathrm{~m}, 2 \mathrm{H}$, Ar-H), 7.47-7.43 (m, $2 \mathrm{H}, \mathrm{Ar}-\mathrm{H}, \mathrm{Ar}-\mathrm{H}), 7.39$ ( $\left.\mathrm{m}, 2 \mathrm{H}, \mathrm{Ar}-\mathrm{H}_{\text {Diast }}\right), 7.33$ (m, $\left.2 \mathrm{H}, \mathrm{Ar}-\mathrm{H}, \mathrm{Ar}-\mathrm{H}_{\text {Diast }}\right), 7.30-7.25$ (m, $\left.4 \mathrm{H}, \mathrm{Ar}-\mathrm{H}, \mathrm{Ar}-\mathrm{H}_{\text {Diast }}\right), 7.24-7.19$ (m, $\left.4 \mathrm{H}, \mathrm{Ar}-\mathrm{H}, \mathrm{Ar}-\mathrm{H}_{\text {Diast }}\right), 7.17$ ( $\left.\mathrm{m}, 1 \mathrm{H}, \mathrm{Ar}-\mathrm{H}_{\text {Diast }}\right), 7.11$ (m, $\left.1 \mathrm{H}, \mathrm{Ar}-\mathrm{H}\right), 5.75$ (dd, $J=10.1,7.8 \mathrm{~Hz}, 1 \mathrm{H}, \mathrm{CHOAc}_{\text {Diast }}$ ), 5.68 (dd, $J=9.9,7.1 \mathrm{~Hz}, 1 \mathrm{H}, \mathrm{CHO}-$ Ac), 5.19 (s, $1 \mathrm{H}, \mathrm{CHAr}_{\text {Diast }}$ ), 4.95 (s, $\left.1 \mathrm{H}, \mathrm{CHAr}\right), 3.99$ (t, J = 9.7 Hz, $1 \mathrm{H}$, $\mathrm{SCHH}), 3.75$ (dd, $\left.J=10.6,7.8 \mathrm{~Hz}, 1 \mathrm{H}, \mathrm{SCHH}_{\text {Diast }}\right), 3.44(\mathrm{dd}, J=9.5,7.1$ 
$\mathrm{Hz}, 1 \mathrm{H}, \mathrm{SCH} H), 3.23\left(\mathrm{t}, J=10.3 \mathrm{~Hz}, 1 \mathrm{H}, \mathrm{SCH} H_{\text {Diast }}\right), 3.12(\mathrm{~m}, 1 \mathrm{H}$, $\left.\mathrm{CH}\left(\mathrm{CH}_{3}\right)_{2 \text { Diast }}\right), 2.91\left(\mathrm{~m}, 1 \mathrm{H}, \mathrm{CH}\left(\mathrm{CH}_{3}\right)_{2}\right), 1.98\left(\mathrm{~s}, 3 \mathrm{H}, \mathrm{CH}_{3}\right), 1.96(\mathrm{~s}, 3 \mathrm{H}$, $\left.\mathrm{CH}_{3 \text { Diast }}\right), 1.43$ (d, $\left.J=6.8 \mathrm{~Hz}, 3 \mathrm{H}, \mathrm{CH}_{3}\left({ }^{i} \mathrm{Pr}\right)\right), 1.31\left(\mathrm{~d}, J=6.8 \mathrm{~Hz}, 3 \mathrm{H}, \mathrm{CH}_{3}\right.$ ( ${ }^{i}$ Pr $\left.)_{\text {Diast }}\right), 1.21\left(\mathrm{~d}, J=6.8 \mathrm{~Hz}, 3 \mathrm{H}, \mathrm{CH}_{3}\left({ }^{(} \mathrm{Pr}\right)\right), 0.80\left(\mathrm{~d}, J=6.7 \mathrm{~Hz}, 3 \mathrm{H}, \mathrm{CH}_{3}\right.$ $\left.\left({ }^{i} \mathrm{Pr}\right)_{\text {Diast }}\right)$.

${ }^{13} \mathrm{C}$ NMR (151 MHz, $\left.\mathrm{CDCl}_{3}\right): \delta=171.0\left(\mathrm{C}=\mathrm{O}_{\text {Diast }}\right), 169.9(\mathrm{C}=\mathrm{O}), 169.1$ $(C=0), 168.7\left(C=O_{\text {Diast }}\right), 165.4\left(C_{q \text { Diast }}\right), 163.6\left(C_{q}\right), 137.7\left(C_{q \text { Diast }}\right), 137.3$ $\left(\mathrm{C}_{\mathrm{q}}\right), 134.0\left(\mathrm{C}_{\mathrm{q} \text { Diast }}\right), 133.8\left(\mathrm{C}_{\mathrm{q}}\right), 128.8\left(2 \mathrm{C}, \mathrm{Ar}-\mathrm{C}_{\text {Diast }}\right), 128.7$ (2 C, Ar-C), 128.6 (2 C, Ar- $C_{\text {Diast }}$ ), 128.5 (2 C, Ar-C), 128.3 (2 C, Ar-C), 127.3 (2 C, Ar- $C_{\text {Diast }}$ ), 125.4 (2 C, Ar- $C_{\text {Diast }}$ ), 125.2 (2 C, Ar-C), 119.3 (2 C, Ar-C), 119.1 (2 C, Ar- $\left.\mathrm{C}_{\text {Diast }}\right), 79.8$ ( $\left.\mathrm{CHOAc}_{\text {Diast }}\right), 76.5$ (CHOAC), 69.5 ( $\mathrm{C}_{\mathrm{q} \text { Diast }}$ ), $67.7\left(\mathrm{C}_{\mathrm{q}}\right), 53.0\left(\mathrm{CHAr}_{\text {Diast }}\right), 49.7(\mathrm{CHAr}), 32.3\left(\mathrm{SCH}_{2}\right.$ Diast $), 30.7\left(\mathrm{SCH}_{2}\right)$, $29.7\left(\mathrm{CH}\left(\mathrm{CH}_{3}\right)_{2}\right.$ Diast $), 27.2\left(\mathrm{CH}\left(\mathrm{CH}_{3}\right)_{2}\right), 22.8\left(\mathrm{CH}_{3}\right.$ Diast $), 22.3\left(\mathrm{CH}_{3}\right), 20.8$ (2 C, $\mathrm{CH}\left(\mathrm{CH}_{3}\right)_{2}$ Diast $), 20.6\left(2 \mathrm{C}, \mathrm{CH}\left(\mathrm{CH}_{3}\right)_{2}\right)$.

MS (EI, $70 \mathrm{eV}): m / z(\%)=408.1(3)[\mathrm{M}]^{+}, 348.1(13)[\mathrm{M}-\mathrm{HOAc}]^{+}, 291.2$ (67) $\left[\mathrm{M}-\mathrm{C}_{4} \mathrm{H}_{5} \mathrm{O}_{2} \mathrm{~S}\right]^{+}$.

HRMS (ESI): $m / z$ [M $+\mathrm{Na}]^{+}$calcd for $\mathrm{C}_{23} \mathrm{H}_{24} \mathrm{~N}_{2} \mathrm{O}_{3} \mathrm{SNa}$ : 409.1580; found: 409.1573.

\section{1-tert-Butyl-4-oxo-3,6-diphenyl-7-thia-2,3-diazaspiro[4.4]non-1- en-9-yl Acetate (3p)}

Yield: $44 \mathrm{mg}$ (42\%); orange solid; $\mathrm{mp} 155-157^{\circ} \mathrm{C} ; R_{f}=0.70$ ( $n$-pentane-Et $\left.{ }_{2} \mathrm{O}, 1: 1\right)$.

IR (ATR): 2965, 1721, 1596, 1490, 1373, 1218, 1044, 967, 849, $748 \mathrm{~cm}^{-1}$. ${ }^{1} \mathrm{H} \mathrm{NMR}\left(600 \mathrm{MHz}, \mathrm{CDCl}_{3}\right): \delta=7.45(\mathrm{~m}, 4 \mathrm{H}, \mathrm{Ar}-\mathrm{H}), 7.28-7.24(\mathrm{~m}, 2 \mathrm{H}$, Ar-H), 7.21 (m, 2 H, Ar-H), 7.17-7.12 (m, $1 \mathrm{H}, \mathrm{Ar}-\mathrm{H}), 7.09$ (m, $1 \mathrm{H}, \mathrm{Ar}-$ $\mathrm{H}), 5.91$ (dd, $J=9.7,7.2 \mathrm{~Hz}, 1 \mathrm{H}, \mathrm{CHOAc}), 5.32(\mathrm{~s}, 1 \mathrm{H}, \mathrm{CHAr}), 4.01$ (t, $J=$ $9.6 \mathrm{~Hz}, 1 \mathrm{H}, \mathrm{SCHH}$ ), 3.43 (dd, J = 9.5, 7.2 Hz, $1 \mathrm{H}, \mathrm{SCHH}$ ), 1.98 (s, $3 \mathrm{H}$, $\left.\mathrm{CH}_{3}\right), 1.47\left(\mathrm{~s}, 9 \mathrm{H},{ }^{t} \mathrm{Bu}\right)$.

${ }^{13} \mathrm{C}$ NMR (151 MHz, $\left.\mathrm{CDCl}_{3}\right): \delta=170.0(\mathrm{C}=0), 168.9(\mathrm{C}=0), 163.6\left(\mathrm{C}_{\mathrm{q}}\right)$, $137.1\left(\mathrm{C}_{\mathrm{q}}\right), 134.3\left(\mathrm{C}_{\mathrm{q}}\right), 128.5(\mathrm{Ar}-\mathrm{C}), 128.5$ (2 C, Ar-C), 128.4 (2 C, Ar-C), 128.1 (2 C, Ar-C), 125.2 (Ar-C), 119.2 (2 C, Ar-C), 77.0 (CHOAC), 68.4 $\left(\mathrm{C}_{\mathrm{q}}\right), 48.5(\mathrm{CHAr}), 37.1\left(\mathrm{C}\left(\mathrm{CH}_{3}\right)_{3}\right), 30.5\left(\mathrm{SCH}_{2}\right), 29.8\left(3 \mathrm{C},{ }^{\mathrm{t}} \mathrm{Bu}\right), 20.7$ $\left(\mathrm{CH}_{3}\right)$.

MS (EI, $70 \mathrm{eV}): m / z(\%)=423.2(36)[\mathrm{M}+\mathrm{H}]^{+}, 422.2(18)[\mathrm{M}]^{+}, 362.1$ (89) $[\mathrm{M}-\mathrm{HOAc}]^{+}, 305.0(100)\left[\mathrm{M}-\mathrm{C}_{4} \mathrm{H}_{5} \mathrm{O}_{2} \mathrm{~S}\right]^{+}$.

HRMS (ESI): $m / z[\mathrm{M}+\mathrm{H}]^{+}$calcd for $\mathrm{C}_{24} \mathrm{H}_{27} \mathrm{~N}_{2} \mathrm{O}_{3} \mathrm{~S}: 423.1737$; found: 423.1736 .

\section{1-Ethyl-4-oxo-3,6-diphenyl-7-thia-2,3-diazaspiro[4.4]non-1-en-9- yl Acetate (3q)}

Yield: $74 \mathrm{mg}$ (76\%); colorless oil; $R_{f}=0.65$ ( $n$-pentane-Et ${ }_{2} \mathrm{O}, 1: 1$ ).

IR (ATR): 2978, 2940, 2106, 1751, 1708, 1596, 1495, 1453, 1353, 1218 , $1142,1049,957,913,837,800,757,728,696 \mathrm{~cm}^{-1}$.

${ }^{1} \mathrm{H}$ NMR $\left(600 \mathrm{MHz}, \mathrm{CDCl}_{3}\right): \delta=7.74(\mathrm{~m}, 2 \mathrm{H}, \mathrm{Ar}-\mathrm{H}), 7.39-7.31(\mathrm{~m}, 5 \mathrm{H}$, Ar-H), 7.24-7.20 (m, 3 H, Ar-H), 5.73 (t, J = 8.6 Hz, $1 \mathrm{H}, \mathrm{CHOAc}), 5.13$ (s, $1 \mathrm{H}, \mathrm{CHAr}$ ), 3.79 (dd, $J=11.0,8.2 \mathrm{~Hz}, 1 \mathrm{H}, \mathrm{SCHH}), 3.17$ (dd, $J=10.9$, 9.1 Hz, $1 \mathrm{H}, \mathrm{SCHH}), 2.68-2.58$ (m, $\left.2 \mathrm{H}, \mathrm{CH}_{2} \mathrm{CH} 3\right), 1.97$ (s, $\left.3 \mathrm{H}, \mathrm{CH}_{3}\right), 1.12$ ( $\mathrm{t}, J=7.2 \mathrm{~Hz}, 3 \mathrm{H}, \mathrm{CH}_{2} \mathrm{CH}_{3}$ ).

${ }^{13} \mathrm{C}$ NMR (151 MHz, $\left.\mathrm{CDCl}_{3}\right): \delta=171.0(\mathrm{C}=0), 169.3(\mathrm{C}=0), 161.3\left(\mathrm{C}_{\mathrm{q}}\right)$, $137.6\left(\mathrm{C}_{\mathrm{q}}\right), 133.3\left(\mathrm{C}_{\mathrm{q}}\right), 128.8(2 \mathrm{C}, \mathrm{Ar}-\mathrm{C}), 128.7(\mathrm{Ar}-\mathrm{C}), 128.5(2 \mathrm{C}, \mathrm{Ar}-\mathrm{C})$, 127.2 (Ar-C), 125.4 (2 C, Ar-C), 119.1 (2 C, Ar-C), 79.3 (CHOAC), 69.2 $\left(\mathrm{C}_{\mathrm{q}}\right), 54.2$ ( $\left.\mathrm{CHAr}\right), 33.1\left(\mathrm{SCH}_{2}\right), 24.5\left(\mathrm{CH}_{2}\right), 20.7\left(\mathrm{CH}_{3}\right), 9.0\left(\mathrm{CH}_{2} \mathrm{CH}_{3}\right)$.

MS (EI, $70 \mathrm{eV}): m / z(\%)=394.0(3)[\mathrm{M}]^{+}, 333.9(12)[\mathrm{M}-\mathrm{HOAC}]^{+}, 77.2$ (100) $\left[\mathrm{C}_{6} \mathrm{H}_{5}\right]^{+}$.

HRMS (ESI): $m / z$ [M + Na] $]^{+}$calcd for $\mathrm{C}_{22} \mathrm{H}_{22} \mathrm{~N}_{2} \mathrm{O}_{3} \mathrm{SNa}$ : 417.1243; found: 417.1242 .

\section{Acknowledgment}

Financial support from the European Research Council (ERC Advanced Grant 320493 'DOMINOCAT') is gratefully acknowledged.

\section{Supporting Information}

Supporting information for this article is available online at http://dx.doi.org/10.1055/s-0035-1562473.

\section{References}

(1) (a) Ohtsuka, T.; Kotaki, H.; Nakayama, N.; Itezono, Y.; Shimma, N.; Kudoh, T.; Kuwahara, T.; Arisawa, M.; Yokose, K. J. Antibiot. 1993, 46, 11. (b) Yoshimura, Y.; Watanabe, M.; Satoh, H.; Ashida, N.; Ijichi, K.; Sakata, S.; Machida, H.; Matsuda, A. J. Med. Chem. 1997, 40, 2177. (c) Yoshikawa, M.; Murakami, T.; Yashiro, K.; Matsuda, H. Chem. Pharm. Bull. 1998, 46, 1339. (d) Wirsching, J.; Voss, J.; Adiwidjaja, G.; Giesler, A.; Kopf, J. Eur. J. Org. Chem. 2001, 1077. (e) Yoshikawa, M.; Morikawa, T.; Matsuda, H.; Tanabe, G.; Muraoka, O. Bioorg. Med. Chem. 2002, 10, 1547.

(2) (a) De Clercq, P. J. Chem. Rev. 1997, 97, 1755. (b) Zempleni, J.; Wijeratne, S. S. K.; Hassan, Y. I. Biofactors 2009, 35, 36.

(3) Johnson, J. W.; Evanoff, D. P.; Savard, M. E.; Lange, G.; Ramadhar, T. R.; Assoud, A.; Taylor, N. J.; Dmitrienko, G. I. J. Org. Chem. 2008, 73, 6970.

(4) For selected examples, see: (a) Zea, A.; Alba, A.-N. R.; Mazzanti, A.; Moyano, A.; Rios, R. Org. Biomol. Chem. 2011, 9, 6519. (b) Liu, L.; Zhong, Y.; Zhang, P.; Jiang, X.; Wang, R. J. Org. Chem. 2012, 77, 10228. (c) Zhang, J.-X.; Li, N.-K.; Liu, Z.-M.; Huang, X.-F.; Geng, Z.-C.; Wang, X.-W. Adv. Synth. Catal. 2013, 355, 797. (d) Liang, J.; Chen, Q.; Liu, L.; Jiang, X.; Wang, R. Org. Biomol. Chem. 2013, 11, 1441. (e) Chauhan, P.; Mahajan, S.; Loh, C. C. J.; Raabe, G.; Enders, D. Org. Lett. 2014, 16, 2954. (f) Han, B.; Huang, W.; Ren, W.; He, G.; Wang, J.-H.; Peng, C. Adv. Synth. Catal. 2015, 357, 561. (g) Li, J.-H.; Du, D.-M. Chem. Asian J. 2014, 9, 3278. (h) Hack, D.; Dürr, A. B.; Deckers, K.; Chauhan, P.; Seling, N.; Rübenach, L.; Mertens, L.; Raabe, G.; Schoenebeck, F.; Enders, D. Angew. Chem. Int. Ed. 2016, 55, 1797.

(5) Chande, M. S.; Barve, P. A.; Suryanarayan, V. J. Heterocycl. Chem. 2007, 44, 49.

(6) (a) Schlemminger, I.; Schmidt, B.; Flockerzi, D.; Tenor, H.; Zitt, C.; Hatzelmann, A.; Marx, D.; Braun, C.; Kuelzer, R.; Heuser, A.; Kley, H. P.; Sterk, G. J. (Nycomed GmbH, Germany) WO2010055083, 2010. (b) Schmidt, B.; Scheufler, C.; Volz, J.; Feth, M. P.; Hummel, R. P.; Hatzelmann, A.; Zitt, C.; Wohlsen, A.; Marx, D.; Kley, H. P.; Ockert, D.; Heuser, A.; Christiaans, J. A. M.; Sterk, G. J.; Menge, W. M. P. B. (Nycomed GmbH, Germany) WO2008138939, 2008.

(7) (a) Zhang, Y.; Wu, S.; Wang, S.; Fang, K.; Dong, G.; Liu, N.; Miao, Z.; Yao, J.; Li, J.; Zhang, W.; Sheng, C.; Wang, W. Eur. J. Org. Chem. 2015, 2013. (b) Wu, S.; Li, Y.; Xu, G.; Chen, S.; Zhang, Y.; Liu, N.; Dong, G.; Miao, C.; Su, H.; Zhang, W.; Sheng, C. Eur. J. Med. Chem. 2016, 115, 141.

(8) Mandha, S. R.; Siliveri, S.; Alla, M.; Bommena, V. R.; Bommineni, M. R.; Balasubramanian, S. Bioorg. Med. Chem. 2012, 22, 5272.

(9) For a feature article, see: Chauhan, P.; Mahajan, S.; Enders, D. Chem. Commun. 2015, 51, 12890.

(10) For a recent review, see: (a) Chauhan, P.; Mahajan, S.; Enders, D. Chem. Rev. 2014, 114, 8807. For selected examples, see: (b) Barco, A.; Baricordi, N.; Benetti, S.; De Risi, C.; Pollini, G. P. 
Tetrahedron Lett. 2006, 47, 8087. (c) Ling, J.-B.; Su, Y.; Zhu, H.-L.; Wang, G.-Y.; Xu, P.-F. Org. Lett. 2012, 14, 1090. (d) Baricordi, N.; Benetti, S.; Bertolasi, V.; De Risi, C.; Pollini, G. P.; Zamberlan, F.; Zanirato, V. Tetrahedron 2012, 68, 208. (e) Su, Y.; Ling, J.-B.; Zhang, S.; Xu, P.-F. J. Org. Chem. 2013, 78, 11053. (f) Xu, C.; Du, J.; Ma, L.; Li, G.; Tao, M.; Zhang, W. Tetrahedron 2013, 69, 4749. (g) Kumar, S. V.; Prasanna, P.; Perumal, S. Tetrahedron Lett. 2013, 54, 6651. (h) Liang, J.-J.; Pan, J.-Y.; Xu, D.-C.; Xie, J.-W. Tetrahedron Lett. 2014, 55, 6335. (i) Bharkavi, C.; Kumar, S. V.; Perumal, S. Synlett 2015, 26, 1665.

(11) (a) Duan, S.-W.; Li, Y.; Liu, Y.-Y.; Zou, Y.-Q.; Shi, D.-Q.; Xiao, W.-J. Chem. Commun. 2012, 48, 5160. (b) Zhao, B.-L.; Liu, L.; Du, D.-M. Eur.J. Org. Chem. 2014, 7850. (c) Zhou, P.; Cai, Y.; Lin, L.; Lian, X.; Xia, Y.; Liu, X.; Feng, X. Adv. Synth. Catal. 2015, 357, 695. (d) Hu, Y.-J.; Wang, X.-B.; Li, S.-Y.; Xie, S.-S.; Wang, K. D. G.; Kong, L.-Y. Tetrahedron Lett. 2015, 56, 105. (e) Mahajan, S.; Chauhan, P.; Blümel, M.; Puttreddy, R.; Rissanen, K.; Raabe, G.; Enders, D. Synthesis 2016, 48, 1131 .
(12) (a) Alemán, J.; Parra, A.; Jiang, H.; Jørgensen, K. A. Chem. Eur. J. 2011, 17, 6890. (b) Storer, R. I.; Aciro, C.; Jones, L. H. Chem. Soc. Rev. 2011, 40, 2330. (c) Ni, X.; Li, X.; Wang, Z.; Cheng, J.-P. Org. Lett. 2014, 16, 1786. (d) Chauhan, P.; Mahajan, S.; Kaya, U.; Hack, D.; Enders, D. Adv. Synth. Catal. 2015, 357, 253.

(13) (a) Takemoto, Y. Chem. Pharm. Bull. 2010, 58, 593. (b) Serdyuk, O. V.; Heckel, C. M.; Tsogoeva, S. B. Org. Biomol. Chem. 2013, 11, 7051. (c) Aitken, L. S.; Arezki, N. R.; Dell'Isola, A.; Cobb, A. J. A. Synthesis 2013, 45, 2627.

(14) CCDC 1484545 (3i) contains the supplementary crystallographic data for this paper. These data can be obtained free of charge from The Cambridge Crystallographic Data Centre via www.ccdc.cam.ac.uk/data_request/cif.

(15) Chen, Q.; Liang, J.; Wang, S.; Wang, D.; Wang, R. Chem. Commun. 2013, 49, 1657 . 